Aus der Abteilung Klinische Neurophysiologie

(Prof. Dr. med. W. Paulus)

im Zentrum Neurologische Medizin

der Medizinischen Fakultät der Universität Göttingen

\title{
Verstärkung des antinozizeptiven Effekts der \\ kathodalen transkraniellen Gleichstromstimulation \\ durch den Dopaminagonisten Pergolid
}

\author{
INAUGURAL-DISSERTATION \\ zur Erlangung des Doktorgrades \\ der Medizinischen Fakultät \\ der Georg-August-Universität zu Göttingen \\ vorgelegt von \\ Inga Bergmann \\ aus Detmold
}

Göttingen 2009 
Dekan: Prof. Dr. med. C. Frömmel

1. Berichterstatterin: PD Dr. rer. nat. A. Antal

2. Berichterstatter/in:

3. Berichterstatter/in:

Tag der mündlichen Prüfung: 
Die Ergebnisse dieser Arbeit wurden veröffentlicht in:

Terney D, Bergmann I, Poreisz C, Chaieb L, Boros K, Nitsche MA, Paulus W, Antal A (2008):

Pergolide increases the efficacy of cathodal direct current stimulation to reduce the amplitude of laser-evoked potentials in humans.

Journal of pain and symptom managment $36,79-91$ 
Inhalt

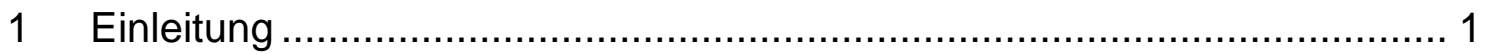

1.1 Im Kontext anderer Experimente/ Historische Grundlagen ................. 2

1.1.1 Wirkmechanismen der tDCS …............................................. 5

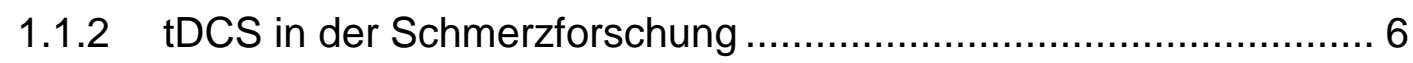

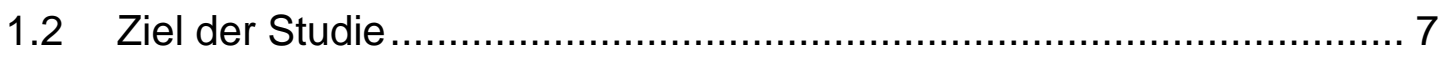

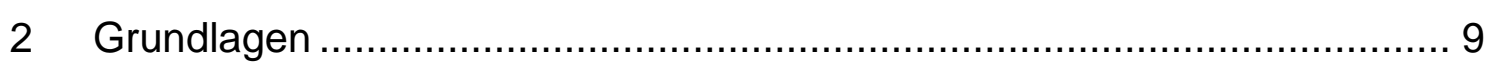

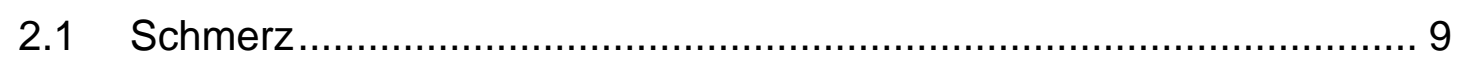

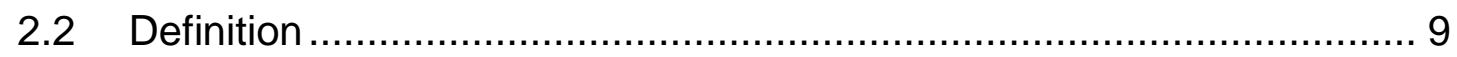

2.2.1 Schmerzmessung .......................................................... 10

2.2.2 Aufbau der peripheren Nozizeption ....................................... 11

2.2.3 Weiterleitung Richtung Gehirn.......................................... 11

2.2.4 Kortikale Repräsentation von Schmerz ............................... 13

2.2.5 Akuter Schmerz .......................................................... 16

2.3 Transkranielle Gleichstromstimulation ..................................... 17

2.4 Langzeitpotenzierung und Langzeitdepression ............................ 19

2.5 Dopamin/ Pergolid ................................................................. 20

2.6 Laser-evozierte Potenziale (LEP) …........................................ 22

2.7 Transkranielle Magnetstimulation ............................................... 25

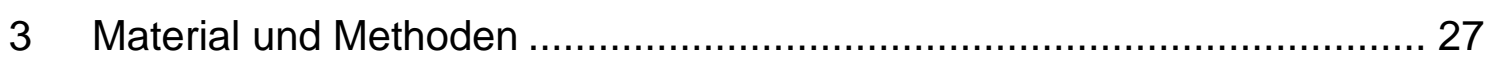

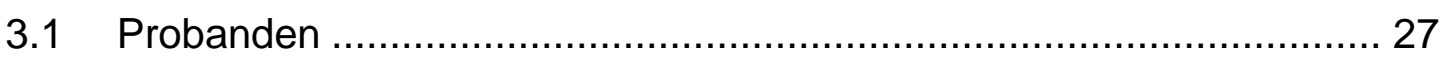

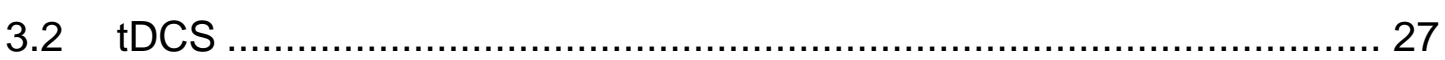

3.3 Laserstimulation ................................................................. 28

3.4 Medikamente ....................................................................... 29

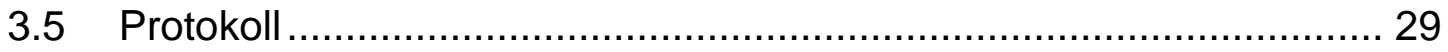

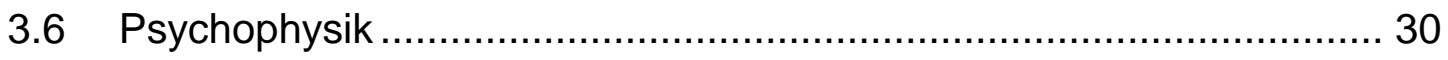

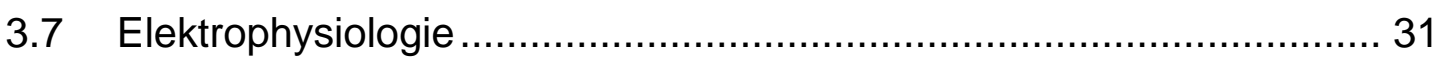

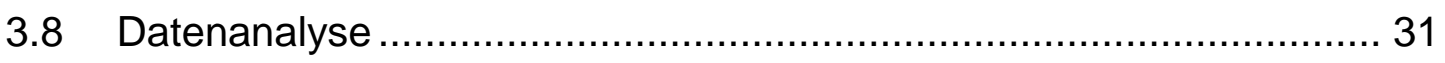

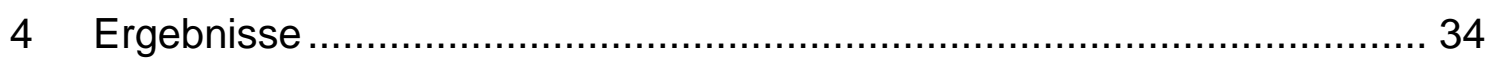

$4.1 \quad$ Laserintensitäten.................................................................. 34

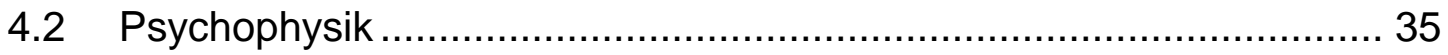

4.3 Elektrophysiologie ............................................................... 37

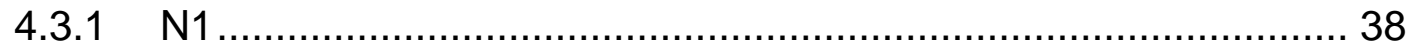

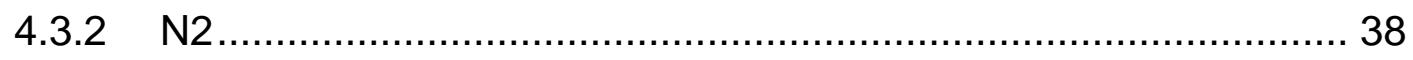

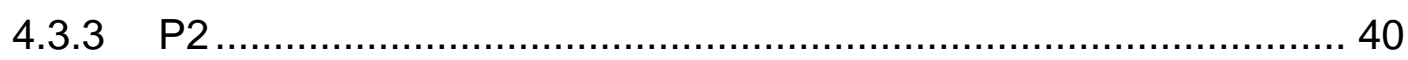




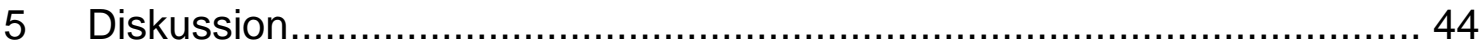

5.1 Stimulation des Motorkortex...................................................... 45

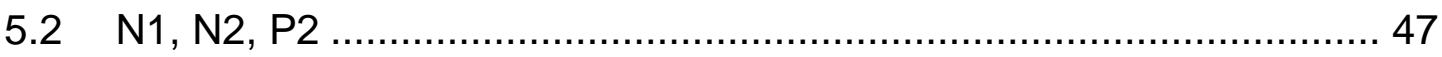

5.3 Sicherheit und mögliche Anwendungsgebiete der Methode .............. 48

5.4 Verlängerung der Wirkung der tDCS durch Pergolid ....................... 50

6 Zusammenfassung und Ausblick .............................................. 53

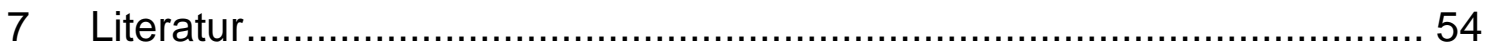

8 Tabellen- und Abbildungsverzeichnis ............................................ 64

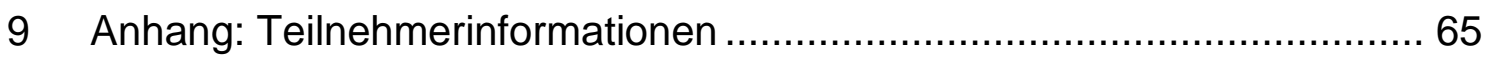




\section{Abkürzungsverzeichnis:}

Abb:

ACC:

ANOVA:

BOLD fMRI:
Abbildung

anterior cingulate cortex (vorderer zingulärer Kortex)

Analysis of Variance (Varianzanalyse)

Bloog Oxygenation Level-Dependent functional Magnetic Resonance Imaging (Blutoxygenierungsabhängige funktionelle Magnetresonanztomographie

Cz: $\quad$ Elektrodenposition nach 10-20-System

D1: Dopamin-1 (Rezeptor)

D2: Dopamin-2 (Rezeptor)

EEG: $\quad$ Elektroenzephalogramm

$E P$ : evoziertes Potenzial

EPSP: exzitatorisches postsynaptisches Potenzial fMRT: funktionelle Magnetresonanztomographie

Fz: Elektrodenposition nach 10-20-System

LEP: Laser-evoziertes Potenzial

LTD: $\quad$ Long-term-depression (Langzeitdepression)

LTP: $\quad$ Long-term-potentiation (Langzeitpotenzierung)

M1: $\quad$ primärer Motorkortex

MEP: muskulär evoziertes Potenzial

N1: $\quad$ erstes negatives Potenzial

N2: $\quad$ zweites negatives Potenzial

$\mathrm{NaCl}: \quad$ Natrium-Chlorid

NAS: $\quad$ Numerische Analogskala

NMDA: N-Methyl-D-Aspartat

P2:

PET:

zweite positive Amplitude

Positronen-Emissions-Tomographie

$\mathrm{Pz}$ :

rCBF:

Elektrodenposition nach 10-20-System

regional Cerebral Blood Flow (regionaler zerebraler Blutfluss)

S1: $\quad$ primärer somatosensorischer Kortex

S2: sekundärer somatosensorischer Kortex 
T3:

Elektrodenposition nach 10-20-System

T4:

Elektrodenposition nach 10-20-System

tDCS:

transcranial Direct Current Stimulation (transkranielle

Gleichstromstimulation)

TMS: $\quad$ Transkranielle Magnetstimulation

ZNS: $\quad$ zentrales Nervensystem 


\section{Einleitung}

Schmerzbehandlung spielt im klinischen Alltag in fast allen Disziplinen der medizinischen Versorgung eine große Rolle und ist häufig mit großen Schwierigkeiten wie Medikamentenunverträglichkeiten, Neben- und Wechselwirkungen und Wirkverlusten im Verlauf einer Therapie verbunden. Deshalb stellt sich immer die Frage nach neuen, gut verträglichen und durchführbaren Möglichkeiten der Schmerztherapie.

Das Thema meiner Arbeit ist die Beeinflussung der akuten Schmerzwahrnehmung durch kathodale transkranielle Gleichstromstimulation und die Verlängerung der Wirkdauer dieser Methode durch den Dopaminagonisten Pergolid. Die Gleichstromstimulation (= transcranial direct current stimulation, tDCS) ist eine Methode zur non-invasiven Stromstimulation des Gehirns und hat sich in letzter Zeit in vielen Untersuchungen als vielversprechende Methode zur Behandlung von Schmerzen unter Beweis gestellt. In ersten Experimenten wird sie bereits an Patienten mit zum Bespiel chronischen Rückenschmerzen angewandt (Fregni et al. 2006 a).

Schwacher Gleichstrom, das heißt, elektrischer Stromfluss in eine konstante Richtung (Priori 2003), kann die Exzitabilität des Kortex verändern, wobei anodale Stimulation die Exzitabilität erhöht und kathodale diese erniedrigt (Nitsche und Paulus 2000, 2001). In einem vorausgegangenen Experiment zeigte sich, dass kathodale Stromstimulation die Schmerzwahrnehmung herabsetzen kann (Csifcsak et al. 2009). Wir bewerteten die Wirkung der kathodalen tDCS einerseits anhand von subjektiven Schmerzskalen, andererseits benutzten wir als objektiven Faktor Laser-evozierte Potenziale. Unser Experiment zeigt, dass eine fünfzehnminütige kathodale Stromstimulation über dem primären Motorkortex die subjektive Schmerzwahrnehmung und die N2Komponente der Laser-evozierten Potenziale signifikant senken kann. Durch die Einnahme des Dopaminagonisten Pergolid wird die Dauer dieser Wirkung signifikant verlängert. Die Verminderung der subjektiven 
Schmerzwahrnehmung bleibt bis zu vierzig Minuten erhalten, und die N2Komponente kann für bis zu vierundzwanzig Stunden gesenkt werden. Bevor die tDCS sich als reguläre therapeutische Methode etablieren lässt, muss allerdings noch viel geforscht werden. Mit unserem Experiment möchten wir überprüfen, ob die Methode sich durch die Anwendung von Pergolid verbessern lässt. Wir gehen davon aus, dass die Einnahme des Dopaminagonisten eine Verlängerung der Nachwirkungen der tDCS bewirken kann. Dadurch könnte die Methode für eine eventuelle klinische Anwendung verbessert werden.

\subsection{Im Kontext anderer Experimente/ Historische Grundlagen}

Versuche mit Stromstimulationen des Gehirns reichen weit in die Geschichte der Medizin zurück: schon 40 vor Christus beobachtete der römische Arzt Scribonius Largus, dass Kopfschmerzen durch Auflegen eines Zitterrochens gebessert werden konnten. Plinius der Ältere und der griechische Arzt Galen (ca. 129-216 n. Chr.) machten ähnliche Beobachtungen (umfassende historische Übersicht: Priori 2003).

Die ersten für uns richtungsweisenden Versuche wurden an Tieren durchgeführt. Seit den 50er Jahren des vergangenen Jahrhunderts wurden zunächst an Ratten, Katzen und Kaninchen Experimente mit Stromstimulation der Gehirnoberfläche durchgeführt. Dazu wurde ein Loch in die Schädeldecke gebohrt, um die Elektroden auf der Dura mater zu platzieren und so direkt das Gehirn zu stimulieren.

Mit verschiedenen Methoden zur Untersuchung der Auswirkungen der Stimulation wurden unterschiedliche Beobachtungen zusammengetragen:

L. Bindman veröffentlichte 1964 einen Artikel im Journal of Physiology, in dem sie die Auswirkung transkortikaler Polarisierung auf Rattengehirne beschrieb. In dieser Studie wurden evozierte Potenziale durch die Stimulation verändert, und zwar beobachtete man eine Erniedrigung der positiven und eine Erhöhung der negativen Amplitude 
im Elektroenzephalogramm (EEG) bei einer positiven Polarisierung des Kortex (Bindman et al. 1964). Eine weitere wichtige Beobachtung war, dass diese Effekte auch über das Stimulationsende hinaus anhielten, wenn man mindestens einige Minuten stimulierte.

An Katzengehirnen wurden ähnlich Beobachtungen gemacht: Eine Stimulation des Motorkortex resultiert entweder in einer Inhibition oder einer Verstärkung der neuronalen Aktivität, wobei eine Positivierung des Motorkortex zu einer Aktivierung der neuronalen Aktivität führt (Creutzfeld et al. 1962).

O. Lippold beschrieb 1964 einen Versuch mit transkranieller Stimulation am Menschen. Er gab an, mit Elektroden über der Augenbraue und am Bein der Probanden Einfluss auf deren Gemütslage nehmen zu können. Laut Lippold wirkte sich anodale transkranielle Stimulation positiv, kathodale negativ auf die Psyche der Patienten aus. Nach anodaler Stimulation seien die Patienten gut gelaunt und leistungsfähig, kathodale Stimulation führe zu Müdigkeit und schlechter Laune. Nach diesen Beobachtungen wandten Lippold und seine Kollegen die anodale tDCS auch bei depressiven Patienten an. Sie beobachteten nach mehrwöchiger (mind. 14 Tage) Stimulation eine Besserung des Gemütszustandes der Patienten. Es sei also möglich, mit Stimulation des Gehirns Einfluss auf die Psyche der Menschen zu nehmen. Die von Lippold beschriebenen signifikanten Ergebnisse konnten in anderen Studien allerdings nicht reproduziert werden (Sheffield und Mowbray 1968).

Die beobachteten Veränderungen, also die neuronale Aktivierung durch anodale Stimulation und die Abschwächung evozierter Potenziale durch kathodale Stimulation, werden durch eine Veränderung des Membranpotenzials hervorgerufen. Je nach Flussrichtung des Stroms wird das Ruhemembranpotenzial verstärkt oder verringert, so dass die Entladungsrate der Neurone entsprechend verstärkt oder abgeschwächt wird (Purpura und McMurty 1964). 
Verallgemeinernd kann man aus diesen Versuchen also den Schluss ziehen, dass anodale Stimulation zu einer Erhöhung der Erregbarkeit der Neurone führt, und kathodale Stimulation die Erregbarkeit vermindert. Diese Ergebnisse waren die Grundlage für unsere Untersuchung der Wirkung der kathodalen Stimulation auf die Schmerzwahrnehmung.

Seit einigen Jahren ist die Stromstimulation jetzt wieder in den Blickpunkt verschiedener Forscher gerückt. An der Universität Göttingen werden seit dem Jahr 1999 Versuche mit tDCS durchgeführt. In der ersten Arbeit aus dem Jahr 2000 beschrieben Nitsche und Paulus erstmals durch tDCS hervorgerufene Veränderungen und Nacheffekte der Stimulation am Menschen, nachgewiesen durch transkranielle Magnetstimulation (TMS) (Nitsche und Paulus 2000). Es folgten verschiedene Studien, in denen die tDCS als Methode weiter etabliert und erforscht wurde:

Man fand heraus, dass es möglich ist, die Erregbarkeitserhöhung des Motorkortex für einige Zeit nach Stimulation aufrecht zu erhalten (wieder mit TMS getestet). Die Dauer der tDCS korrelierte hier mit der Dauer der Nacheffekte (Nitsche und Paulus 2001). Die Veränderungen im Kortex durch Gleichstromstimulation wurden auch im fMRT (BOLD MRI) nachgewiesen: kathodale tDCS führte zu einer Erniedrigung der neuronalen Aktivität, und auch eine tendenzielle Erhöhung der Aktivität durch anodale Stimulation konnte festgestellt werden (Baudewig et al. 2001).

Nach neun Minuten kathodaler Stimulation können per TMS signifikante Nachwirkungen von bis zu einer Stunde Dauer nachgewiesen werden, unter neun Minuten Stimulationsdauer endet die Reduktion der neuronalen Erregbarkeit wenige Minuten nach Stimulation (Nitsche et al. 2003 c). Eine längere Wirklatenz nach entsprechend längerer Stimulationsdauer wurde auch schon 1964 von Bindman et al. beobachtet. 


\subsubsection{Wirkmechanismen der tDCS}

Um die zugrunde liegenden Mechanismen der Aktivitätsveränderung zu verstehen, blockierten Liebetanz und Nitsche in mehreren Experimenten selektiv lonenkanäle und Transmitter-Rezeptoren, um deren Einfluss auf die tDCS zu erfahren: der Natriumkanalblocker Carbamazepin und der NMDA-Rezeptor-Antagonist Dextromethorphan veränderten die von reiner tDCS hervorgerufenen Effekte auf die Neuroplastizität, weshalb auf eine Beteiligung dieser Rezeptoren bzw. Kanäle geschlossen wurde. Durch die Antagonisierung der NMDA-Rezeptoren mit Dextromethorphan wurde die Wirkung sowohl der anodalen als auch der kathodalen Stimulation abgeschwächt. Somit wird vermutet, dass die NMDARezeptoren eine entscheidende Rolle bei der Entstehung der tDCSEffekte spielen (Nitsche et al. 2003 b, 2004, 2006, Liebetanz et al. 2002). Die Blockierung der Natriumkanäle durch Carbamazepin verhinderte nur die Wirkung der anodalen Stimulation. Carbamazepin stabilisiert spannungsabhängig das Membranpotenzial, und da unter diesem Medikament die sonst beobachteten Folgen der anodalen tDCS ausblieben, wurde gefolgert, dass eine Voraussetzung für die Wirkung der anodalen tDCS ein veränderliches Membranpotenzial (die Fähigkeit zur Depolarisation) ist (Liebetanz et al. 2002).

Eine weitere Studie zur Auswirkung der verschiedenen lonenkanäle und damit der Membranpotenziale und des NMDA-Rezeptors auf die Entstehung von Nacheffekten durch tDCS folgte 2003 von Nitsche et al. Es wurden wieder Carbamazepin und Dextromethorphan eingesetzt, außerdem der Kalziumkanalblocker Flunarizin. Die Medikamente wurden zu kürzer und länger andauernder anodaler und kathodaler tDCS verabreicht, um den Einfluss sowohl auf direkte als auch auf Nacheffekte der Stimulation zu beurteilen. Carbamazepin und Flunarizin verhinderten die anodale, aktivitätssteigernde Wirkung der tDCS, Dextromethorphan bewirkte sowohl bei anodaler als auch bei kathodaler Stimulation das Ausbleiben lang anhaltender Nachwirkungen der tDCS, die Resultate der kurzen tDCS wurden allerdings nicht verändert. Diese Studie ließ den 
wichtigen Schluss zu, dass die lang anhaltenden Nachwirkungen von der Modulation der NMDA-Rezeptoren abhängen (Nitsche et al. 2003 b). Ein weiterer Hinweis auf die Abhängigkeit der Nachwirkungen der tDCS vom NMDA-Rezeptor folgt aus der Erkenntnis, dass sie unter Medikation mit Katecholaminen (Amphetamin) länger anhalten, allerdings NMDARezeptor-abhängig (Nitsche et al. 2004).

Diese Abhängigkeit der tDCS-Nachwirkungen wird vermutlich dopaminerg unterstützt. Eine Studie zeigte, dass die medikamentöse Blockierung des D2-Rezeptors mit Sulpirid die Entstehung von Nachwirkungen der tDCS nahezu völlig verhinderte (Nitsche et al. 2006). Es wird angenommen, dass die Nacheffekte der tDCS Gemeinsamkeiten mit solchen Veränderungen des ZNS haben, die durch Langzeitpotenzierung (LTP) und Langzeitdepression (LTD) hervorgerufen werden. LTPs entstehen durch NMDA-RezeptorAktivierung, deren Blockade, wie oben beschrieben, die Entstehung von Nacheffekten der tDCS verhindert (Paulus 2004).

\subsection{2 tDCS in der Schmerzforschung}

Stromstimulation des Gehirns hat auch einen Einfluss auf die Schmerzwahrnehmung. Die Stimulation des primären Motorkortex hat eine Erleichterung sowohl chronischer Schmerzen als auch experimentell zugefügter Schmerzen zur Folge (Hirayama et al. 2006; Tsubokawa et al. 1993; Lende et al. 1971; Csifcsak et al. 2009). So fand Lende heraus, dass chronischer Schmerz durch die Resektion des präzentralen und korrespondierenden postzentralen Gyrus erleichtert werden kann; Tsubokawa et al. implantierten Elektroden zur dauerhaften Stimulation des primären Motorkortex $M 1$ und erreichte dadurch eine Schmerzlinderung bei Patienten mit Phantomschmerzen.

Hirayama und Saitoh verglichen die Wirkung von drei verschiedenen Stimulationsorten: im Gegensatz zu einer TMS über S1 bzw. dem 
prämotorischen Areal zeigte die M1-Stimulation eine signifikante Besserung für Patienten mit Phantomschmerz.

Lefaucheur et al. führten 2001 zum ersten Mal einen Versuch zur Linderung chronischer Schmerzen mittels rTMS des Motorkortex durch und erreichten eine vorübergehende Schmerzminderung von bis zu 12 Tagen (Lefaucheur et al. 2001, Fregni et al. 2007).

Tsubokawa et al. beschrieben die schmerzlindernde Wirkung von Motorkortexstimulation bei Patienten mit thalamischem Schmerzsyndrom (Tsubokawa et al. 1991).

Es wird vermutet, dass die schmerzsenkende Wirkung der Motorkortexstimulation auf einem Anstieg des Blutflusses im ipsilateralen ventrolateralen Thalamus durch die dort vorherrschenden Verbindungen zum Motor- und Prämotorkortex beruht. Das Ausmaß der Schmerzsenkung korreliert außerdem mit gesteigertem Blutfluss im Gyrus cinguli (Brown 2001).

\subsection{Ziel der Studie}

Entscheidend für die Fragestellung meiner Arbeit waren die Ergebnisse, die Csifcsak et al. 2009 vorlegten und die bereits weiter oben erwähnte Studie über die Unterstützung der tDCS-induzierten Nacheffekte durch Dopamin (Nitsche et al. 2006).

Csifcsak et al. verglichen in ihrer Studie die Effekte kathodaler Stimulation auf die Schmerzwahrnehmung mit denen der anodalen. Wie in unserem Experiment wurde die Wahrnehmung zum einen elektrophysiologisch, zum anderen subjektiv durch numerische Analogskalen bewertet, die Schmerzreize wurden mit einem Laser induziert. Die kathodale Stimulation des primären Motorkortex bewirkte eine signifikante Reduktion der Schmerzwahrnehmung und eine signifikante Verkleinerung der N2 und P2 Komponenten der LEP.

Der Einfluss von Dopamin auf die tDCS wurde 2006 von Nitsche et al. untersucht: 
Dopamin unterstützt und verlängert die durch tDCS über dem Motorkortex hervorgerufene Neuroplastizität. Ebenso wie durch die Blockierung der NMDA-Rezeptoren wird auch durch eine D2-RezeptorBlockade das Auftreten von Nacheffekten verhindert, die Agonisierung der D2- und D1-Rezeptoren durch Pergolid aber bewirkt eine Verlängerung der durch kathodale Stimulation hervorgerufenen Aktivitätsminderung. Diese Änderung der Erregbarkeit, die durch Muskelevozierte Potenziale (MEPs) nachgewiesen wurde, deutet auf einen ursächlichen Zusammenhang zwischen dem dopaminergen System und der Induktion und Aufrechterhaltung von Neuroplastizität, also neuronaler Veränderlichkeit (Nitsche et al. 2006).

Unser Ziel war es herauszufinden, inwiefern der Dopaminagonist Pergolid die durch kathodale Stromstimulation hervorgerufene Senkung der Schmerzwahrnehmung verlängern und unterstützen kann. Grundlegend für unseren Versuchsansatz war, dass

1. kathodale Gleichstromstimulation über dem primären Motorkortex die neuronale Erregbarkeit hemmt (Nitsche und Paulus 2000, 2001);

2. kathodale Gleichstromstimulation über dem primären Motorkortex die akute Schmerzwahrnehmung von gesunden Probanden senken kann (Csifcsak et al. 2009) und

3. die Nachwirkungen der Gleichstromstimulation durch die Gabe von Dopaminagonisten länger anhaltend waren (Nitsche et al. 2006). Unsere Frage war also:

„Kann die schmerzsenkende Wirkung der kathodalen Gleichstromstimulation durch einen Dopaminagonisten verlängert werden und kann dieses mit objektiven Methoden dokumentiert werden?" 


\section{Grundlagen}

\section{$2.1 \quad \underline{\text { Schmerz }}$}

2.2 Definition

„Schmerz ist eine unangenehme, mit tatsächlichem oder potenziellem Gewebeschaden assoziierte sensorische und emotionale Erfahrung. Sie geht in der Regel mit dem Verlangen nach Beendigung und künftiger Meidung der auslösenden Reize einher und kann durch kognitive Faktoren wie Aufmerksamkeit oder vorangegangene Erfahrungen moduliert werden. Dieses Zusammenspiel von sensorischen, affektiven und kognitiven Faktoren und seine essenzielle Bedeutung für die Erhaltung der physischen Unversehrtheit wie auch für das krankhafte Erleben unterscheiden Schmerz wesentlich von anderen Modalitäten." (Ploner und Schnitzler 2004, S. 962)

Schmerzwahrnehmung gehört zur protopathischen Sensibilität.

Sie wird in verschiedene Wahrnehmungsarten eingeteilt, nämlich in den ersten und zweiten Schmerz. Hierbei handelt es sich um zeitlich versetzte Wahrnehmungen des Schmerzes. Der erste Schmerz ist stechend, kurz und gut lokalisierbar. Er wird über die schnell leitenden Adelta-Fasern geleitet und hat eine Reaktionszeit von etwa 400-500ms. Der zweite Schmerz wird über C-Fasern geleitet, dauert länger an, ist eher brennend und schlechter lokalisierbar. Die Reaktionszeit beträgt ca. 1000ms nach Schmerzstimulation der Hand (Ploner et al. 2002).

Die primäre Schmerzwahrnehmung setzt sich aus der sensorischdiskriminativen, der affektiven (emotionalen), der motorischen, der kognitiven und der vegetativen, autonomen Komponente zusammen (Schaible und Schmidt 2007). Diese Komponenten sind gemeinsam für die Schmerzwahrnehmung verantwortlich, verfügen allerdings über unterschiedliche Verarbeitungswege. 
Eine weitere Einteilung kann nach dem Entstehungsort vorgenommen werden. Der somatische Oberflächenschmerz entsteht durch Hautreizung, ist gut lokalisierbar und klingt nach Reizende schnell wieder ab. Der somatische Tiefenschmerz entsteht in Knochen, Muskeln, Gelenken oder Bindegewebe, ist eher dumpf und schlechter lokalisierbar. Viszeraler Tiefenschmerz hat seinen Ursprung in den inneren Organen, kann dumpf und schlecht lokalisierbar oder kolikartig sein.

\subsubsection{Schmerzmessung}

Schmerz kann subjektiv und objektiv gemessen werden. Zur subjektiven Messung gehört auch die von uns verwendete psychophysikalische Methode. Der Schmerz wird vom Probanden/Patienten eingeschätzt und dann subjektiv mit Hilfe einer Skala bewertet, zum Beispiel anhand einer visuellen Analogskala (VAS) oder, wie in unserem Experiment, einer numerischen Analogskala (z.B. $1=$ Wärmeempfindung, 2 bis $10=$ schwacher bis sehr starker Schmerz). Schmerz kann aber auch objektiv gemessen werden, beispielsweise durch die Aufzeichnung vegetativer Funktionen oder, wie in unserem Fall, die Aufzeichnung von evozierten Potenzialen. 


\subsubsection{Aufbau der peripheren Nozizeption}

Die erste Instanz bei der Wahrnehmung des Oberflächenschmerzes sind die peripheren Nozizeptoren in der Haut. Sie liegen als sog. freie Nervenendigungen im Gewebe, das heißt, dass die Axone der Schmerzafferenzen in feinen Verzweigungen enden, die nur noch dünn myelinisiert sind. In diesen sensorischen Endigungen findet die Reiztransduktion statt, das heißt, der noxische Reiz wird in elektrische Erregung/ Aktionspotenziale umgewandelt.

Die Zellkörper der Nozizeptoren liegen in den Spinalganglien der Rückenmarkssegmente. Von dort werden die Reize über die Hinterwurzel ins Rückenmark geleitet.

Die Schmerzwahrnehmung erfolgt hauptsächlich über die unmyelinisierten Fasern der Gruppe C/ Gruppe IV, die langsamer leiten (Leitungsgeschwindigkeit um $1 \mathrm{~m} / \mathrm{sek}$ ), zu einem geringen Teil auch über die dünn myelinisierten Fasern der Gruppe A-delta/ III (Leitungsgeschwindigkeit 2,5-30m/sek) (Schaible und Schmidt 2007). Über A-delta/ III werden mechanischer und thermal-mechanischer Schmerz geleitet (erster Schmerz: scharfer, stechender Schmerz und brennender Schmerz, verantwortlich für schnelle nozizeptive Vorgänge wie Schutzreflexe). Thermisch- mechanischer Schmerz, also Hitze- und Kälteschmerz, wird sowohl über Fasern der Gruppe III als auch IV geleitet, polymodaler Schmerz (zweiter Schmerz: langsamer, brennender Schmerz) wird ausschließlich über Fasern der Gruppe IV geleitet. (Basbaum und Jessel 2000)

\subsubsection{Weiterleitung Richtung Gehirn}

Die Information über Schmerz wird vom Spinalganglion aus über die Hinterwurzeln ins Rückenmark geleitet. Dort geschieht dann die erste Umschaltung, und zwar durch Projektionsneurone im dorsalen Horn. 
(Das dorsale Horn ist in mehrere Laminae unterteilt, in denen jeweils unterschiedliche Qualitäten empfangen werden. Zum Beispiel in Lamina I A-delta nozizeptive Fasern; Lamina II enthält Interneurone, die zur Transmission der C-Faser-Informationen dienen.)

Einmal im Rückenmark geschieht die Schmerzweiterleitung Richtung Gehirn dann über fünf Stränge: den Tractus spinothalamicus, Tractus spinoreticularis, Tractus spinomesencephalus, Tractus spinohypothalamicus und Tractus cervicothalamicus.

Tractus spinothalamicus und spinoreticularis bilden gemeinsam die Vorderseitenstrangbahn, die im Rückenmark auf die kontralaterale Seite kreuzt. Sie ist die wichtigste Bahn der Schmerzwahrnehmung (Schaible und Schmidt 2007)

Im Tractus spinothalamicus werden die Neurone aus Lamina I und V-VII gesammelt. Sie ziehen bis in den Thalamus, der eine wichtige Rolle in der Wahrnehmung des Schmerzes spielt. . Da er direkt in supraspinale autonome Kontrollzentren projiziert, wird inm die Aktivierung komplexer neuroendokriner und kardiovaskulärer Reaktionen zugeschrieben.

Im Tractus spinoreticularis treffen die Axone aus Lamina VII und VIII aufeinander. Er endet im Thalamus und in der Formatio reticularis.

Der Tractus spinomesencephalicus enthält die Axone aus Laminae I und V. Er endet ebenfalls in der Formatio reticularis und außerdem in der grauen Substanz rund um das Aquädukt. Außerdem projiziert er über den Tractus spinobrachialis zu den parabrachialen Nuklei, die dann weiter zur Amygdala und damit ins limbische System projizieren. Somit ist der Tractus spinomesencephalicus auch für die emotionale Wahrnehmung des Schmerzes zuständig.

Der Tractus cervicothalamicus entsteht aus Neuronen der Laminae III und IV. Die meisten Axone kreuzen auf die Gegenseite und steigen dann im Lemniscus medialis des Hirnstamms auf. Sie enden zumeist im Thalamus. 


\subsubsection{Kortikale Repräsentation von Schmerz}

Schmerzwahrnehmung ist ein komplizierter, noch nicht vollständig verstandener Prozess. Verschiedenste Hirnareale sind daran beteiligt, man geht von einer sogenannte "Schmerzmatrix" aus, in die die bisher bekannten Areale der Schmerzverarbeitung involviert sind (Peyron et al. 2000)

Im Thalamus sind verschiedene Kerngruppen an der Schmerzverarbeitung beteiligt. Die wichtigste Rolle spielen die lateralen und die medialen Kerne. Der Tractus spinothalamicus projiziert in die lateralen Nuklei, bestehend aus Nucleus ventralis posteromedialis, dem Nucleus ventralis posterolateralis, und dem Nucleus lateralis posterior. Die Neurone dieser Nuklei haben kleine rezeptive Felder, entsprechend den in sie projizierenden Rückenmarksneuronen. Deshalb wird vermutet, dass der laterale Thalamus hauptsächlich für die Lokalisation der Schmerzsensationen zuständig ist, der so vermittelte Schmerz wird als akuter Schmerz wahrgenommen.

Verletzungen des Tractus spinothalamicus, z. B. durch Infarkte, resultieren im so genannten zentralen oder auch thalamischen Schmerz, Dysästhesien, einem abnormen und starken Schmerzempfinden.

Die medialen Thalamuskerne erhalten ihre Afferenzen hauptsächlich aus den Laminae VII und VIII des Hinterhorns. Dieser Leitungspfad wird aufgrund seiner frühen Entwicklung in der Evolution der Säugetiere auch als Tractus paleospinothalamicus bezeichnet. Er enthält auch Afferenzen aus der Formatio reticularis des Hirnstamms. Die Neurone des medialen Thalamus gehören teilweise zum so genannten unspezifischen Thalamus. Als solche reagieren sie auf noxische Stimuli, projizieren aber auch in die Basalganglien und viele andere kortikale Gebiete. Es wird innen daher nicht nur eine Funktion in der Schmerzverarbeitung zugeschrieben, sondern auch die Verarbeitung unspezifischer Stimuli, die eine „Arousal“-Reaktion hervorrufen. 
Die weitere Projektion der Schmerzwahrnehmung kann zum Beispiel mit Hilfe der PET (Positron Emission Tomography), fMRI (functional Magnetic Resonance Imaging), im Elektroenzephalogramm (EEG) oder in der Magnetenzephalographie(MEG) dargestellt werden. Es zeigt sich, dass Schmerzwahrnehmung vom Thalamus sehr komplex über den ganzen Kortex verteilt projiziert wird. Man findet Antworten auf Stimulation der Nozizeptoren im somatosensorischen Kortex, im Gyrus cinguli, und im Inselortex. Auf die Funktion der verschiedenen Kortexareale kann man auch durch Beobachtungen von Patienten mit Läsionen in einzelnen Gebieten schließen.

Ploner und Schnitzler (2004) fassten die neusten Erkenntnisse auf dem Gebiet der kortikalen Repräsentation von Schmerz zusammen:

Die diskriminativen Fähigkeiten der Schmerzwahrnehmung sind hiernach dem primären somatosensorischen Kortex (S1) zuzuordnen, der im Gyrus postcentralis lokalisiert ist. Er erhält Afferenzen aus dem Nucleus ventralis posterolateralis. Für die diskriminative Funktion spricht, dass die dort befindlichen, auf Schmerz reagierenden Neurone somatotopisch angeordnet sind, kleine rezeptive Felder haben, und Dauer und Intensität der Schmerzwahrnehmung sich in ihrer Aktivität widerspiegelt. Eine MEG-Studie zeigte, dass die Amplitude der assoziierten S1-Aktivität die Intensität der applizierten Schmerzen sehr genau darstellte (Timmermann et al. 2001). Der S1 scheint wesentlich für die Repräsentation des ersten Schmerzes zu sein.

Der sekundäre somatosensorische Kortex (S2) ist im parietalen Operkulum lokalisiert und vermutlich für die Verarbeitung der kognitiven Komponente des Schmerzes zuständig. Die entsprechenden Afferenzen kommen aus dem Nucleus ventralis posterior inferior. Hier kann man mittels EEG und MEG eindeutige Aktivierung bei Schmerzapplikation feststellen. Entsprechend der kognitiven Aufgabe des S2 besitzt er große, bilaterale rezeptive Felder, und eine kortikale Antwort auf Reize ist nicht von ihrer Intensität abhängig (Aktivierung nach dem Alles-oder-Nichts-Prinzip). Der sekundäre 
somatosensorische Kortex hat Projektionsneurone zur Insel und von dort zu gedächtnisrelevanten Arealen des medialen Temporallappens. S2 könnte somit eine wichtige Funktion bei der Weitergabe nozizeptiver Informationen ins limbische System und somit beim schmerzbezogenen Lernen haben.

Auch der insuläre Kortex ist an der Schmerzverarbeitung beteiligt, wie in verschiedenen Studien durch bildgebende Verfahren und Tierexperimente nachgewiesen wurde (Zusammenfassung: Ploner und Schnitzler 2004). Über die genaue Funktion der Insel herrscht allerdings noch Unklarheit. Die multiplen Verbindungen zu anderen Teilen des Gehirns legen eine supramodale, integrative Funktion der Insel nahe, es bedarf allerdings weiterer Erforschung (Ploner und Schnitzler 2004). Die affektiv- emotionale Verarbeitung des Schmerzes bzw. die Verarbeitung des zweiten Schmerzes wird dem vorderen zingulären Kortex (ACC) zugeschrieben. Er wird über die medialen Thalamuskerne mit nozizeptiven Informationen versorgt. Die Rolle des ACC wurde hauptsächlich in Läsionsstudien untersucht. Nach chirurgischer Läsion des ACC wurde eine Verminderung des Schmerzaffekts und des Vermeidungsverhaltens auf Schmerzreize beobachtet. 


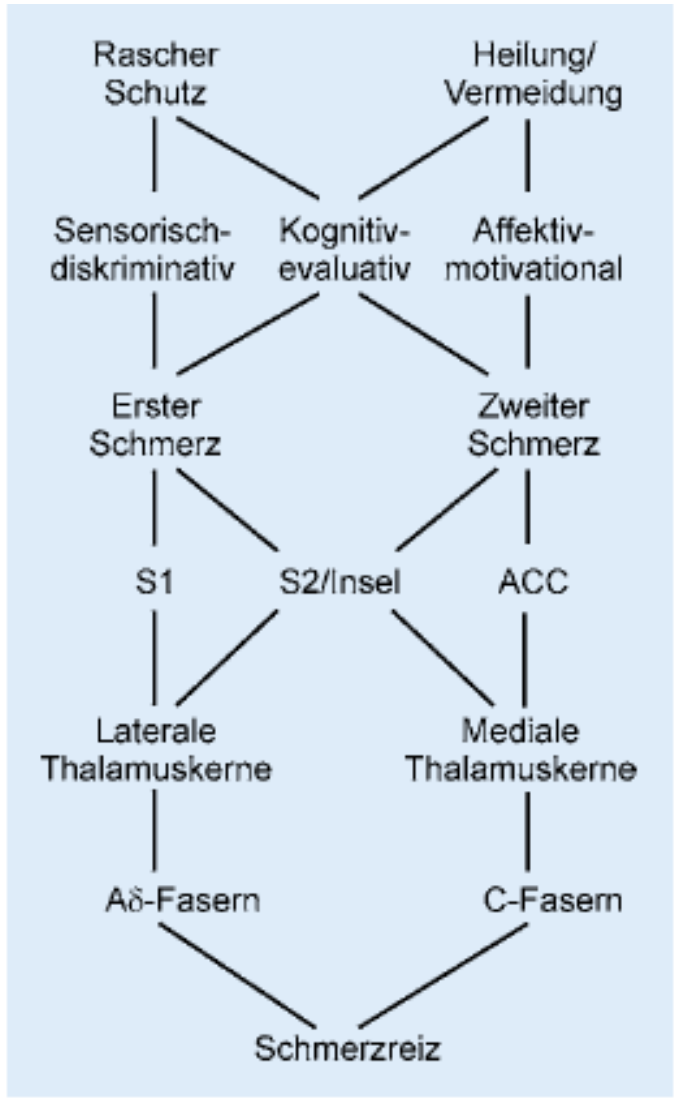

Abbildung 2-1: nach Ploner und Schnitzler 2004, S. 968: die oben beschriebenen Strukturen im vermuteten funktionellen Zusammenhang

\subsection{5 $\quad$ Akuter Schmerz}

Bei dem in unserer Studie durch Laserreize hervorgerufenen Schmerz handelt es sich um akuten Schmerz. Im Gegensatz zum chronischen Schmerz ist der akute Schmerz eine direkte und schnelle Antwort auf unmittelbare Reize. Er dient vor allem dem Schutz vor akuten Gefahren und zieht häufig eine Abwehrreaktion nach sich. Akuter Schmerz ist meist von kurzer Dauer und gut zu behandeln. 


\subsection{Transkranielle Gleichstromstimulation}

Transkranielle Gleichstromstimulation (engl. transcranial direct current stimulation, tDCS) ist eine non- invasive Methode zur Veränderung neuronaler Erregbarkeit. Die Wirksamkeit leichter Stromapplikation auf das Gehirn ist schon lange bekannt, wobei das Gehirn anfangs z. B. intraoperativ direkt stimuliert wurde. Die transkranielle Stimulation ist eine vielversprechende Methode zur Behandlung von z.B. Migräne, neuropathischen Schmerzen oder, wie in unserem Ansatz, zur Minderung akuter Schmerzen und wird gegenwärtig intensiv erforscht. Sie ist natürlich weitaus schonender als invasive Stimulation, nebenwirkungsarm und schmerzfrei (Antal und Paulus 2007, Fregni et al. 2006 a).

TDCS bewirkt nachweisbar eine Veränderung der kortikalen Erregbarkeit. Zunächst wird diese durch eine Veränderung des Ruhemembranpotenzials der Neurone im stimulierten Bereich und vermutlich auch in angrenzenden Bereichen hervorgerufen (Purpura und McMurty 1965, Nitsche et al. 2003 b). Die meisten Neurone feuern, wenn inr Ruhemembranpotenzial depolarisiert und werden durch eine Hyperpolarisation gehemmt. Die tDCS ahmt diese natürlichen Vorgänge nach, kathodale Stimulation wirkt hyperpolarisierend auf die Neuronenmembranen, während anodale Stimulation depolarisierend wirkt (Liebetanz et al. 2002). Kathodale Stimulation ist damit aktivitätsmindernd, während anodale aktivitätssteigernd wirkt (Paulus 2004). (Dies wurde zunächst von Nitsche und Paulus durch die Veränderlichkeit Muskel evozierter Potenziale (MEP) des Daumens durch tDCS gezeigt. Der kontralaterale Motorkortex wurde mit tDCS anodal oder kathodal stimuliert, danach wurden MEPs durch TMS hervorgerufen. Deren Amplitude verhielt sich entsprechend der oben genannten Aktivitätsminderung oder -steigerung je nach Stromart, war also unter anodaler Stimulation vergrößert und unter kathodaler Stimulation vermindert (Nitsche und Paulus 2000).) 
Die Nachwirkungen der tDCS hingegen scheinen von NMDA-Rezeptoren abhängig zu sein: durch pharmakologische Blockierung der NMDARezeptoren werden die Nacheffekte der tDCS erheblich verkürzt (Liebetanz et al. 2002, Nitsche et al. 2003 b). Die Nacheffekte werden, ebenso wie LTP und LTD, durch dopaminerge Mechanismen unterstützt bzw. verlängert. Insbesondere der D2-Rezeptor spielt eine wichtige Rolle bei der Induktion von Nachwirkungen, da eine selektive Blockade dieses Rezeptors Nacheffekte verhinderte (Nitsche et al. 2006).

Die kathodale Minderung und anodale Steigerung der Aktivität lassen sich auch auf die Schmerzwahrnehmung übertragen. Laut Csifcsaks und Antals Studie über modulierende Effekte der tDCS auf die Wahrnehmung akuten Schmerzes minderte kathodale Stimulation des Motorkortex die Wahrnehmung starken, Laser-induzierten Schmerzes. Anodale und Placebostimulation dagegen hatten keinen Einfluss auf die Schmerzwahrnehmung. Dies zeigte sich durch gesteigerte bzw. verminderte Amplituden von Laser-evozierten Potenzialen nach Applikation von tDCS, die kathodale Stimulation reduzierte die Amplituden des evozierten Potenzials (Csifcsak et al. 2009).

Dass der Motorkortex an der Schmerzwahrnehmung beteiligt ist, wurde bereits in mehreren Studien bestätigt (Tsubokawa et al. 1991, 1993, Brown 2001). Dessen Resektion resultierte in lang anhaltender Schmerzfreiheit für chronische Schmerzpatienten (Lende et al. 1971); Tsubokawa beschrieb die schmerzlindernde Wirkung von Motorkortexstimulation beim thalamischen Schmerzsyndrom (Tsubokawa et al. 1993).

Transkranielle Gleichstromstimulation ist also eine Möglichkeit, neuronale Plastizität am Menschen zu induzieren und zu erforschen. Ähnlich wie Langzeitpotenzierung und Langzeitdepression ist auch sie von NMDA-Rezeptoren abhängig und kann in der Wirklatenz durch Dopamin beeinflusst werden (Liebetanz et al. 2002, Nitsche et al. 2006). 


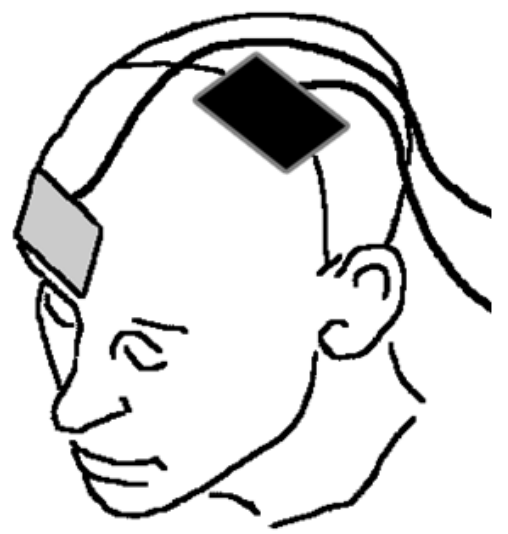

Abbildung 2-2: Platzierung der Elektroden für die tDCS. (nach Lang et al. 2005, S. 496)

\subsection{Langzeitpotenzierung und Langzeitdepression}

Langzeitpotenzierung und Langzeitdepression (engl. Long-timepotentiation, LTP und long-time-depression, LTD) sind Formen neuronaler Plastizität. Sie gelten als Voraussetzung für Lernen und Erinnern, also die Fähigkeit, aufgrund von Erlebtem die Reaktionsweise des Systems auf sinnvolle Art und Weise zu ändern (Birbaumer und Schmidt 2007).

Langzeitpotenzierung wurde erstmals 1966 von Terje Lomo beschrieben (Lomo 1966, 2003). Sie funktioniert über eine hochfrequente Aktivierung glutamaterger Synapsen durch Aktionspotenziale. Ein einzelnes Aktionspotenzial bewirkt zunächst die Öffnung von Non-NMDA-Kanälen mit entsprechendem loneneinstrom und einem schwachen exzitatorischen postsynaptischen Potenzial (EPSP). Eine wiederholte, 
tetanische Stimulierung des präsynaptischen Neurons führt über bestimmte Mechanismen zur zusätzlichen Öffnung von NMDA-Kanälen, durch die dann $\mathrm{Ca}^{2+}$ einströmt. Dies führt dann zum Ziel der LTP: durch die Aktivierung bestimmter zellulärer Enzyme wird die Empfindlichkeit der Synapse zum Beispiel durch den Einbau von neuen Glutamatrezeptoren herabgesetzt und es kommt zu einer vereinfachten Erregbarkeit und somit Nutzbarkeit dieser neuronalen Verbindung (Heckmann und Dudel 2007, Cooke und Bliss 2006).

Langzeitdepression (LTD) wird ebenfalls als ein Mechanismus zur Veränderung neuronaler Plastizität angesehen. Man nimmt an, dass sie durch niederfrequente Aktionspotenziale an der präsynaptischen Membran ausgelöst wird. An der Wirkung sind ebenfalls Calciumionen und Glutamatrezeptoren beteiligt, die über verschiedene intrazelluläre Abläufe zu einer anhaltenden Reduzierung der synaptischen Übertragungsstärke führen (Pape 2005).

\subsection{Dopamin/ Pergolid}

Dopamin ist ein Botenstoff des Gehirns, der eine wichtige Rolle für unsere Wahrnehmung und Emotionen spielt.

Wie bereits oben angedeutet, sind LTP und LTD von NMDA-Rezeptoren abhängig. Diese Mechanismen der synaptischen Plastizität werden unter anderem dopaminerg unterstützt und verfestigt (Jay 2003, Nitsche et al. 2006).

Verschiedene Studien haben sich bereits mit der Rolle des Dopamins, zum Einen in der Neuroplastizität, zum Anderen in der Stabilisierung der durch tDCS hervorgerufenen Veränderungen, befasst. Endogenes Dopamin spielt eine Schlüsselrolle in der Veränderlichkeit und Beeinflussbarkeit von Synapsen. Der vermutete Mechanismus ist in Abb. 2-2 dargestellt (Jay 2003). 
Otani et al. bestätigten einen fördernden Effekt von Dopamin auch auf Langzeitdepression, vermutlich über eine Verstärkung der postsynaptischen Depolarisation während der tetanischen Reizung (Otani et al. 1998).

Die NMDA-Rezeptoren sind also an der Etablierung neuronaler Veränderungen, der Neuroplastizität, beteiligt, die durch LTPs und LTDs hervorgerufen wird. Die Wirkung der NMDA-Rezeptoren in diesem Zusammenhang wird wiederum durch Dopamin beeinflusst. Nitsche fand heraus, dass Dopamin die Etablierung von LTPs und LTDs durch NMDARezeptoren unterstützt. D2-Rezeptor-Aktivierung verstärkte die Bildung von LTPs und spielt damit laut Nitsches Studie eine unterstützende und wichtige Rolle in der Induktion von Neuroplastizität. Dieses fand er durch selektive Blockierung der D1- und D2-Rezeptoren bzw. deren Aktivierung heraus. Veränderte Erregbarkeit wurde in dieser Studie durch tDCS hervorgerufen. Die selektive Blockierung von D2-Rezeptoren verringerte dabei die Nacheffekte der tDCS, Aktivierung der D2-Rezeptoren mit Pergolid jedoch verlängerte die Nacheffekte. Pergolid kann also über die unterstützende Wirkung auf NMDA-Rezeptoren die Nachwirkungen der tDCS verstärken (Nitsche et al. 2006).

Ziemann et al. stellten eine Verbindung zwischen TMS und Dopamin her: ihre Studie zeigte, dass die kortikale Ruhephase (silent period) nach Motorkortexstimulation mit TMS durch die Einnahme des DopaminAgonisten Pergolid verlängert wurde (Ziemann et al. 1996). 


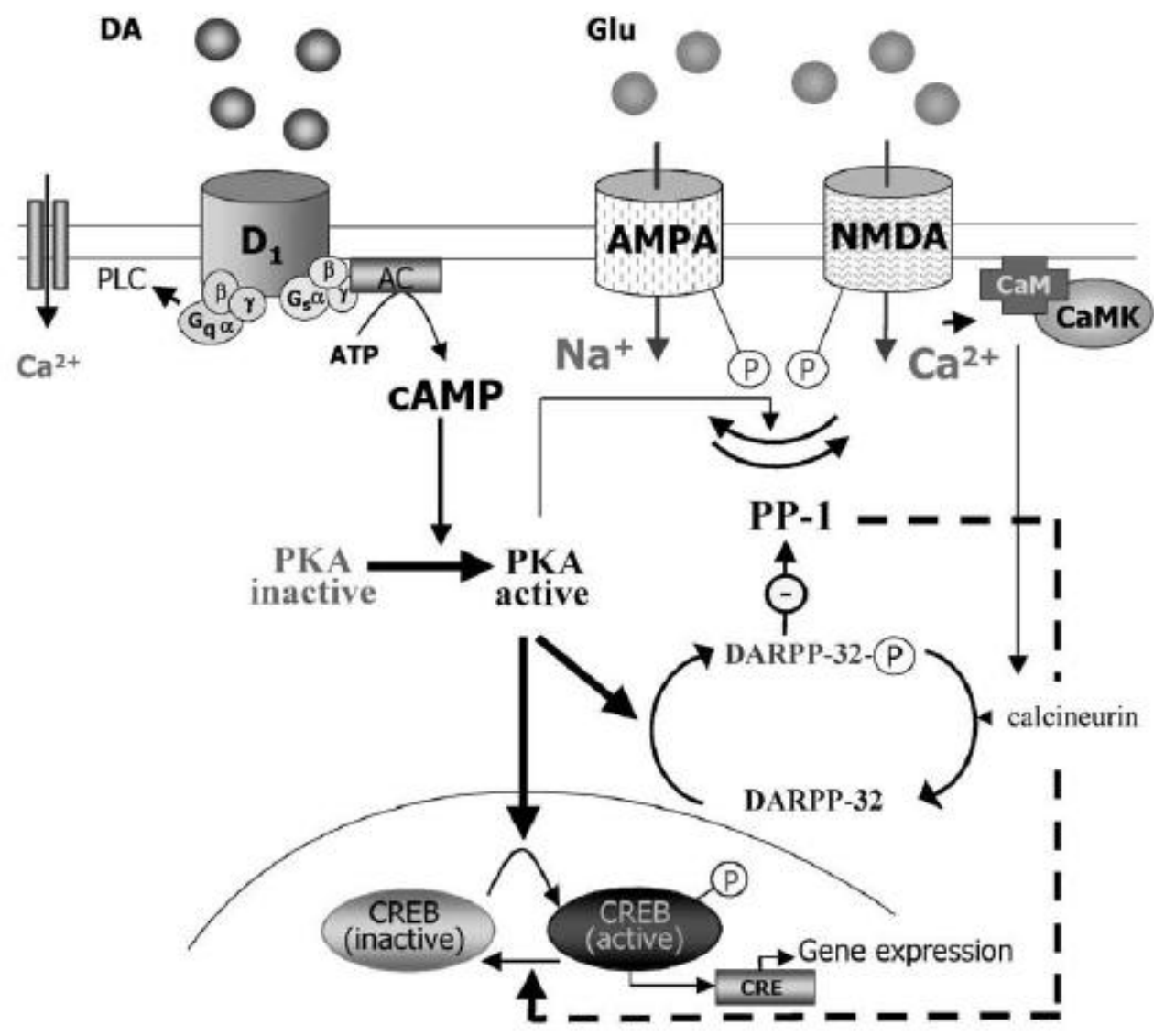

Abbildung 2-3: Wechselwirkung zwischen dem D1-Rezeptor und cAMP/PP-

1/PKA-Pfad bei der Entstehung von LTP (nach Jay 2003, S.382).

\subsection{Laser-evozierte Potenziale (LEP)}

Diejenigen ereigniskorrelierten Potenziale, die sich im ZNS als Antwort auf eine Reizung von Sensoren, von peripheren Nerven, von sensorischen Bahnen oder Kernen registrieren lassen, werden als evozierte Potenziale (EP) bezeichnet. Es gibt visuell, akustisch oder sensorisch evozierte Potenziale, die alle nach dem Prinzip funktionieren, eine kontrolliert zugefügte Reizung des Nervensystems im EEG darzustellen. So können z.B. Läsionen bestimmter Leitungsbahnen diagnostiziert werden. 
Laser-evozierte Potenziale wurden von der European Federation of Neurological Societies als einfachste und verlässlichste Methode zur Untersuchung der Schmerzbahnen (pain pathways) anerkannt (Cruccu et al. 2004). Sie sind eine wichtige Ergänzung zu den herkömmlichen sensorisch evozierten Potenzialen, weil mit ihrer Hilfe selektiv die Schmerzwahrnehmung dargestellt werden kann. Die Stärke der Schmerzempfindung und die leitenden Bahnen können beurteilt werden. Durch den Laser werden selektiv die freien Nervenendigungen der Adelta und C-Fasern der Hautoberfläche gereizt (Bromm und Treede 1983), die LEPs repräsentieren die von diesen Fasern Richtung Gehirn geleiteten Informationen der Nozizeptoren (Plaghki und Mouraux 2005). In der klinischen Anwendung können mit Hilfe Laser-evozierter Potenziale zum Beispiel Läsionen des spinothalamischen Traktes, des lateralen Hirnstammes oder der thalamo-cortikalen Verbindungen untersucht werden (Treede et al. 2003). Die Laserstrahlen des von uns verwendeten Lasers dringen nur 360 Mikrometer in die oberste Hautschicht ein und erreichen somit selektiv die dort befindlichen Adelta- und C-Fasern.

Plaghki und Mouraux haben herausgefunden, dass anhand der Zeitspanne zwischen einem Stimulus und seiner Darstellung im EEG auf die leitende Faserqualität geschlossen werden kann. A-delta-Fasern haben, da sie mehr Myelin besitzen, eine höhere Leitungsgeschwindigkeit als C-Fasern.

Aufgrund ihrer zeitlichen Abfolge, die mit der Leitungsgeschwindigkeit der Fasern korreliert, kann die wahrgenommene und im EEG aufgezeichnete Schmerzsensation den entsprechenden Fasern zugeordnet werden. Man unterscheidet zwischen erstem und zweitem Schmerz. Der erste Schmerz wird von den Probanden als stechender, lokalisierter Schmerz wahrgenommen, der zweite Schmerz als brennender, eher diffuser Schmerz. Der erste Schmerz, der ca. 350 ms nach der Stimulation wahrgenommen wird, kann den A-delta-Fasern zugeordnet werden und entspricht im EEG dem späten LEP. Der zweite, 
ca. 900 ms nach Stimulation, wird über C-Fasern geleitet und entspricht im EEG der ultraspäten LEP-Komponente (Mouraux et al. 2003).

Für unsere Anwendung der LEPs ist der erste Schmerz von größerer Bedeutung, da er im EEG eindeutig identifiziert werden kann. Die ultraspäte LEP-Komponente kann im EEG nur durch selektive C-FaserAktivierung dargestellt werden (Erhitzung nur bis $40^{\circ} \mathrm{C}$; besonders kleine Durchmesser des Laserstrahls; ischämischer Block myelinisierter Fasern; oder durch selektive Blockierung der A-delta-Fasern) (Treede et al. 2003). Das späte LEP hingegen kann im EEG gut identifiziert werden. Mouraux und Plaghki fanden heraus, dass dieses späte LEP mit dem ersten Schmerz korreliert. Man kann einen Komplex aus negativen und positiven Amplituden erkennen, der die Komponenten N1, N2 und P2 beinhaltet. Das späte LEP besteht aus zwei negativen Amplituden: N1 ca. $170 \mathrm{~ms}$, gefolgt von $\mathrm{N} 2$ ca. $210+/-27 \mathrm{~ms}$ nach Impuls, und einer folgenden positiven Amplitude, P2, ca. 380+/- 53 ms nach Impuls (Plaghki und Mouraux 2005). N2 repräsentiert den sensorischdiskrimminativen Prozess, und P2 die kongnitiv-emotioanle Komponente des Schmerzes. Das späte LEP hat sein Maximum über dem Scheitel und wird vom anterioren Gyrus cinguli generiert (Garcia-Larrea 2003).

LEPs erfüllen verschiedene Voraussetzungen:

1. Der vom Laser hervorgerufene Reiz liegt im Millisekundenbereich und ist damit kurz genug, um die evozierten EEG- Wellen im EEG synchron darstellen zu können.

2. Es ist möglich, viele Stimuli hintereinander auszulösen (wir benutzten mind. Vierzig pro Messung)

3. Der Stimulus ist selektiv für nozi- und thermorezeptive Fasern, da die Haut nicht berührt wird und eine gewisse Eindringtiefe nicht überschritten wird.

4. LEPs sind für die Probanden gut verträglich und haben keine Nebenwirkungen.

(Plaghki und Mouraux 2005) 
Aus allen genannten Gründen sind LEPs für unsere Zwecke der ideale Parameter, um einerseits eine objektive Sicht auf die durch tDCS hervorgerufenen neuroplastischen Veränderungen zu ermöglichen und andererseits, um die für uns entscheidende Schmerzwahrnehmung valide anhand von sich verändernden Amplituden einzuschätzen.

Durch den Laser spüren die Probanden einen leicht stechenden Schmerz, in etwa vergleichbar mit einem Nadelstich, und danach den eher brennenden zweiten Schmerz. Insgesamt ist die Untersuchung nicht unangenehmer als die Anwendung herkömmlicher somatisch evozierter Potenziale, bei denen der Reiz durch Strom erfolgt. Eine mögliche unerwünschte Wirkung der Laserstimulation kann eine fokale Rötung der Haut sein, die allerdings nach spätestens 48 Stunden verschwindet.

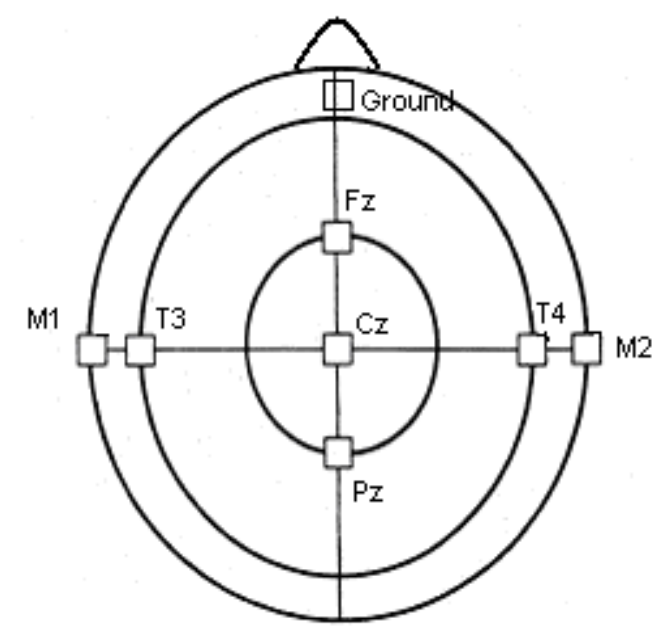

Abbildung 2-4: Positionierung der Elektroden nach dem 10-20- System (nach Plaghki und Mouraux 2005)

\subsection{Transkranielle Magnetstimulation}

Die transkranielle Magnetstimulation (TMS) ist, wie die tDCS, ein Verfahren zur noninvasiven und weitgehend schmerzfreien Hirnstimulation. Mithilfe einer Magnetspule wird ein kurzer 
Magnetstimulus appliziert, der einen Stromfluss im Kortex auslöst und durch das Auslösen axonaler Entladungen einen Impuls an die Peripherie sendet. Die TMS ist hauptsächlich geeignet um oberflächliche, der Kalotte nahe Bezirke des Kortex zu stimulieren, tiefer gelegene Areale werden nach aktuellem Kenntnisstand nur unzureichend erreicht. In unserem Experiment nutzten wir die TMS lediglich zum Auffinden des mit tDCS zu stimulierenden Areals des Motorkortex. Durch Applikation des magnetischen Impulses an entsprechender Stelle kann eine Kontraktion des Musculus abductor digiti minimi ausgelöst werden. 


\section{$3 \quad$ Material und Methoden}

\subsection{Probanden}

An unserer Studie nahmen 15 gesunde Probanden teil und gaben, nachdem sie über alle Aspekte der Untersuchung aufgeklärt wurden, ihr schriftliches Einverständnis. Zwei der Probanden entschieden sich nach der ersten Messung, nicht weiter an der Studie teilzunehmen. Die Ergebnisse eines Probanden wurden aus der Studie ausgeschlossen, weil seine LEPs nicht verlässlich identifiziert werden konnten. Es wurden also die Ergebnisse von 12 Probanden, fünf männlichen und sieben weiblichen, ausgewertet. Sie waren zwischen 23 und 31 Jahren alt. Keiner der Probanden litt an einer chronischen Krankheit. In ihrer Anamnese sind keine psychologischen oder psychiatrischen Erkrankungen bekannt. Außer oraler Kontrazeptiva nahmen sie keine Medikamente ein. Alle Probanden nahmen an einem Durchgang mit Pergolid und einem mit Placebo teil. Außerdem führten wir das Experiment mit sieben Probanden ohne Medikation und tDCS durch.

Die Probanden wurden, nachdem sie über den Versuchsablauf, Risiken usw. aufgeklärt wurden, angewiesen, zwei Stunden vor Beginn der Messung eine Tablette Domperidon bzw. ein Placebo und eineinhalb Stunden vorher eine halbe Tablette Pergolid (entspricht 0,025 mg) bzw. Placebo einzunehmen.

Das Studienprotokoll wurde von der Ethikkommission der Universität Göttingen geprüft und akzeptiert (Nummer 21/3/04) und entspricht der Deklaration von Helsinki.

\section{$3.2 \underline{t D C S}$}

tDCS wurde mittels eines batteriebetriebenen Stimulators der Firma Schneider Elektronik appliziert. Mittels TMS wurde vor dem Experiment das linke Repräsentationsareal des Musculus. abductor digiti minimi 
ausfindig gemacht. Über diesem wurde die kathodale Elektrode, und über der rechten Augenbraue die Referenzelektrode in $5 \times 7 \mathrm{~cm}$ großen Schwämmen platziert und mit Gummibändern befestigt. Diese Platzierung ist nach Nitsche und Paulus (2000) die beste Methode, um eine Veränderung der Erregbarkeit des Motorkortex zu erreichen. Der Gleichstrom wurde mit einer Intensität von $1 \mathrm{~mA}$ über 15 Minuten appliziert.

\subsection{Laserstimulation}

Für die Laserstimulation wurde eine Tm:YAG- Laser (WaveLight Laser Technologie AG, Erlangen) benutzt. Dieser Laser hat eine Eindringtiefe von ca. 360 Mikrometer in die Haut, somit wird das subkutane Gewebe nicht berührt und nur das Gebiet der freien nozizeptiven Endungen erreicht. Die Wellenlänge liegt mit 2000 nm nahe dem infraroten Bereich. Die Dauer eines Impulses beträgt $1 \mathrm{~ms}$, der Durchschnitt des Strahls auf der Haut $7 \mathrm{~mm}$. Er erlaubt eine präzise Bestimmung der applizierten Energie (Treede et al. 2003): Bei allen Messungen wurde ein LaserHandrückenabstand von $30 \mathrm{~cm}$ eingehalten. Auf den Handrücken der Probanden wurden ca. $5 \times 4 \mathrm{~cm}$ große Hautareale markiert. In diesem Bereich wurde der Laserstrahl von Stimulus zu Stimulus ein wenig verschoben, um eine Adaptation zu vermeiden. Vor jeder Messung wurde die Temperatur der Haut gemessen und diese mit einer Wärmelampe erwärmt, wenn sie unter $35^{\circ} \mathrm{C}$ lag.

Vor Beginn der Messungen wurde durch schrittweise Steigerung der Laserenergie, beginnend mit $200 \mathrm{~mJ}$ und einer Steigerung um jeweils 50 mJ, die Wahrnehmungs- bzw. Schmerzschwelle festgestellt. Entsprechend wurde dann während der EEG- Aufnahmen mit der ca. 1,5-1,6fachen Energie der Schmerzschwelle stimuliert. Jede Hand wurde mit 40 Laserimpulsen stimuliert, wobei bei der Hälfte der Probanden mit der linken, bei der anderen Hälfte mit der rechten Hand begonnen wurde. Die einzelnen Stimuli folgten in einem Abstand von ca. 8-15 Sekunden. 
Während der Laserstimulation trugen Versuchsleiter und Probanden Laserschutzbrillen, die Probanden außerdem Ohrenstöpsel um auditorische Artefakte zu vermeiden.

\subsection{Medikamente}

Die Probanden nahmen entweder $0,025 \mathrm{mg}$ Pergolid, einen Dopaminrezeptoragonist (agonisiert am D1- und D2-Rezeptor), und 10 mg Domperidon als Antiemetikum, oder entsprechende Placebos (Glukose), ein. Die Medikamente wurden zwei Stunden vor der Messung eingenommen. Innerhalb dieser Zeit erreichen sie ein stabiles Plasmalevel (Deleu et al. 2002) und zeigen Effekte im ZNS (Ziemann et al. 1996, 1997). Die einzelnen Untersuchungen fanden mit einem Abstand von mindestens einer Woche statt, um kumulative Wirkungen der Medikamente zu verhindern.

\subsection{Protokoll}

Der Versuch wurde doppelblind und randomisiert durchgeführt, das heißt, weder Probanden noch Versuchsleiter wussten, ob als Medikation Placebo oder Pergolid verabreicht wurden. Die Medikation wurde randomisiert ausgegeben.

Zu Beginn des Versuchs wurde zunächst mittels TMS im linken Motorkortex M1 das Areal des Musculus abductor digitus minimus ausfindig gemacht und markiert, dann wurden die EEG-Elektroden für ein fünf-Kanal-EEG nach dem 10-20-System aufgeklebt. Hierauf folgte die erste Messung. Durch zehn Laser-Stimuli auf jedem Handrücken wurde die Schmerzgrenze festgestellt, indem von $200 \mathrm{~mJ}$ in 50erSchritten aufwärts stimuliert wurde. Darauf folgten 40 Stimuli je Hand, wobei bei der Hälfte der Probanden mit der linken, bei der anderen Hälfte mit der rechten Hand begonnen wurde (diese erste Messung ist die 
VOR-Messung). Die LEPs wurden gemessen und zeitgleich die Psychophysik dokumentiert.

Nach diesem ersten Durchgang folgten 15 Minuten tDCS, dann der zweite Durchgang LEP (NACH 1), der dritte folgte 40 Min nach Ende der tDCS (NACH 2), der vierte 2 Stunden danach $(\mathrm{NACH} 3)$ und der fünfte am nächsten Tag 24 Stunden nach Ende der tDCS (NACH 4), jeweils mit Dokumentation der subjektiven Schmerzwahrnehmung.

Mit sieben der Probanden wurde eine dritte Messung ohne tDCS und Medikament durchgeführt, um die normale Habituation (Gewöhnung) einschätzen zu können. Nach längerer Schmerzexposition ist eine Verminderung der Schmerzwahrnehmung durch Gewöhnung physiologisch. Wir wollten feststellen, ob die beobachtete Minderung der Schmerzwahrnehmung unter tDCS und Pergolid stärker ist als durch Habituation.

Zwischen den Messungen wurde ein zeitlicher Abstand von mindestens einer Woche eingehalten, um sicherzugehen, dass die Medikamente in der Zwischenzeit eliminiert wurden.

\subsection{Psychophysik}

Für die subjektive Evaluation der Schmerzereignisse nutzten wir die verbale Numerische Analog Skala (NAS). Die Probanden wurden angewiesen, jede einzelne Schmerzsensation auf einer Skala von 1-10 einzuschätzen ( $1=$ Wärme, $2=$ =eichter Schmerz $-10=$ starker Schmerz), und zwar jeweils 1-2 Sekunden nach Wahrnehmung des Reizes. 


\subsection{Elektrophysiologie}

Für die Aufnahme der Laser-evozierten Potenziale wurde ein FünfKanal-EEG verwendet (BrainAmp system, Brain Products, München). Dieses erlaubt eine sichere Identifizierung der späten LEP-Komponenten N1, N2 und P2 (Treede et al. 2003).

Drei Elektroden wurden nach dem 10-20-System über der Mittellinie des Kopfes platziert, $\mathrm{Fz}, \mathrm{Cz}$ und $\mathrm{Pz}$ (mit den verbundenen Mastoiden als Referenz), und zwei temporal, T3 und T4 (mit Fz als Referenz). Die Impedanz wurde unter $5 \mathrm{kOhm}$ gehalten. Die verbundenen Mastoide bildeten die Referenz, die Erdungs-Elektrode wurde auf der Stirn befestigt. Die Daten wurden mit einer Abtastrate von $1000 \mathrm{~Hz}$ aufgenommen und offline analysiert. Zunächst wurden ein $0,5 \mathrm{~Hz}$ lowcutoff und ein $30 \mathrm{~Hz}$ high-cutoff Filter benutzt.

Nach der automatischen Artefaktentfernung wurden alle vierzig Epochen noch einmal inspiziert und selektiert. Diejenigen mit Artefakten (z.B. durch Bewegung oder Blinzeln) wurden ausgeschlossen. Alle Aufnahmen bestanden aus mindestens 35 Epochen ohne Artefakte. Die Korrektur der Baseline wurde auf der Basis eines prästimulatorischen Intervalls von 100 ms durchgeführt.

\subsection{Datenanalyse}

Um die Amplituden der verschiedenen Probanden zu normen, teilten wir sämtliche $\mathrm{NACH}$-Amplituden, also die, die nach der tDCS aufgenommen wurden, durch die VOR-Werte. So konnten wir die individuell unterschiedlichen Amplituden der Probanden besser vergleichen. Schmerz wird bilateral repräsentiert (Frot und Mauguière 2003, Creach et al. 2000), und unsere Analysen ergaben keine signifikanten Unterschiede zwischen beiden Seiten. Deshalb wurden die Ergebnisse beider Seiten gemittelt und zur Analyse verwendet. 
Die Ergebnisse der numerischen Analogskala, die Werte der N1-, N2-, P2-Amplituden und die der N2-P2-Strecke wurden einzeln gemittelt und in die mehrfaktorielle ANOVA eingegeben. Dabei wurden als Variable die beiden möglichen Medikamente (Placebo oder Pergolid, entspricht

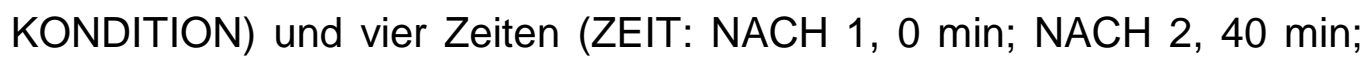
$\mathrm{NACH}$ 3, 2 h; NACH 4, 24 Std) eingegeben, also 2 x KONDITION x 4 x ZEIT. Die Ergebnisse der Kontrollgruppe ohne Medikation wurden ebenfalls genormt und in gleicher Weise in die ANOVA eingegeben $(3 \mathrm{x}$ KONDITION (Placebo oder Pergolid oder Kontrolle).

Um die Wirkung der tDCS separat unter jeder der Bedingungen beurteilen zu können, wurden jeweils univariante ANOVAS der Ergebnisse Pergolid, Placebo und Kontrolle durchgeführt.

Eine signifikante Veränderung wurde angenommen, wenn entweder die KONDITION x ZEIT-Interaktion signifikante Unterschiede aufwies, oder im Zeitverlauf in der univarianten ANOVA signifikante Unterschiede deutlich wurden.

Die Post-hoc-Analyse wurde mit dem Fischer-LSD-Test durchgeführt.

Um die einzelnen Zeitpunkte separat für jedes Individuum beurteilen zu können, wurden die psychophysikalischen Ergebnisse der einzelnen Messungen gemittelt und in einen Students-t-Test eingegeben. 

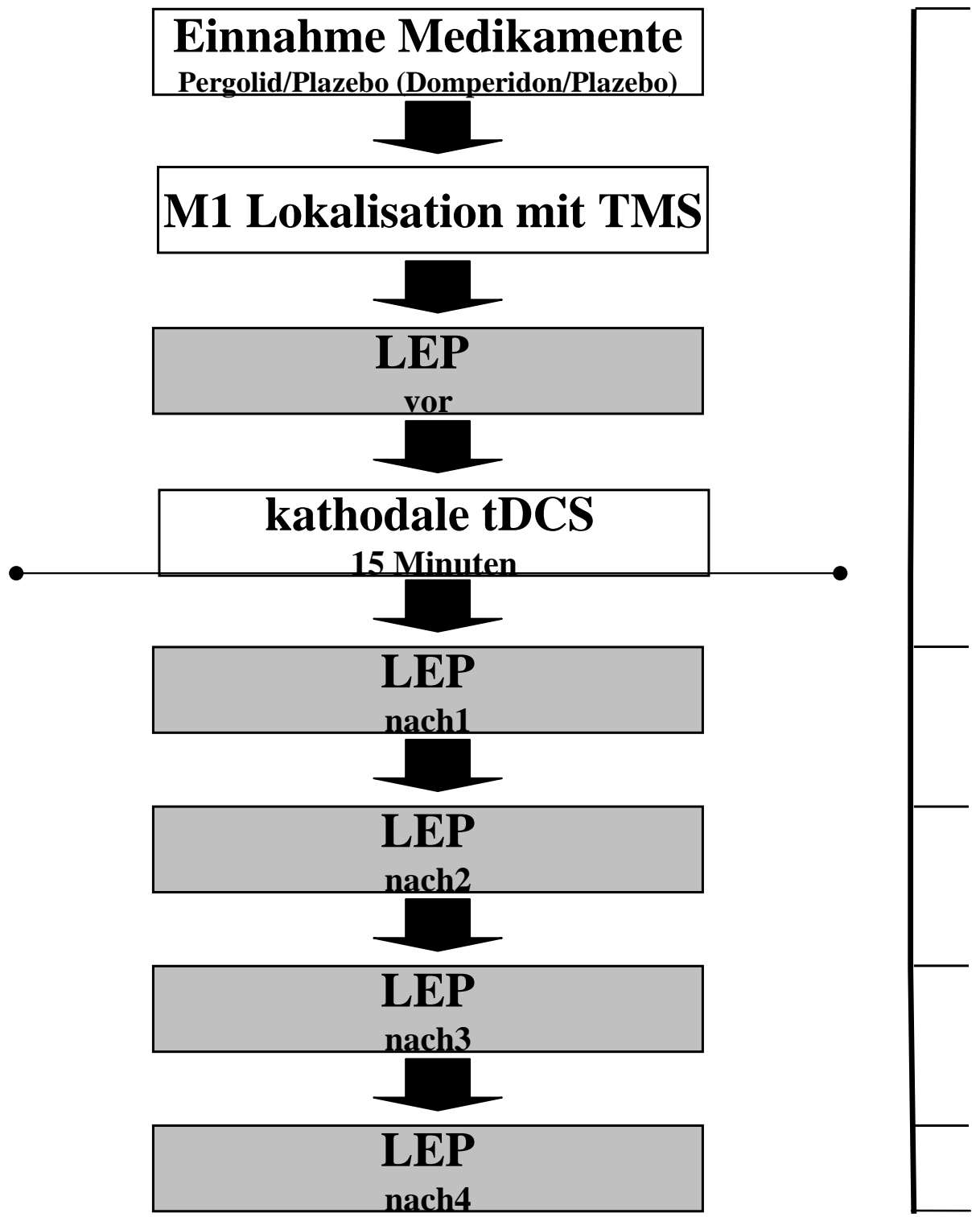

2 Stunden vor tDCS

\section{Min nach tDCS 40 Min nach tDCS}

2 Stunden nach tDCS

24 Stunden nach tDCS

Abbildung 3-1: Fließschema des Versuchsprotokolls 


\section{$4 \quad$ Ergebnisse}

Insgesamt wurde der Versuch von den Probanden gut toleriert. Bis auf gelegentlich leichte Kopfschmerzen nach der Stimulation und ein Jucken unter den Elektroden während der Stimulation zeigten sich keine schweren Unannehmlichkeiten. Übelkeit wurde von keinem der Probanden beklagt.

Kathodale tDCS über dem Motorkortex verminderte die subjektive Schmerzwahrnehmung und die Amplituden der LEPs im Vergleich zur Kontrollgruppe.

Die Veränderung der kortikalen Erregbarkeit hielt nach 15minütiger tDCS bis zu zwei Stunden an.

Durch den Dopaminagonisten Pergolid konnte eine Verlängerung des schmerzmindernden Effekts durch die tDCS für bis zu 24 Stunden erreicht werden.

\subsection{Laserintensitäten}

Alle Probanden verspürten einen stechenden Schmerz durch die Laserimpulse.

Der Vergleich der Laserintensitäten unter Placebo, Pergolid und in der Kontrolle ergab keinen signifikanten Unterschied, die Ergebnisse wurden somit nicht von Unterschieden in der Laserintensität beeinflusst. Bei den Versuchen mit Pergolid ergab sich eine mittlere Laserintensität von 21,32 $\mathrm{mJ} / \mathrm{mm}^{2}$ mit einer Streuung von 19,5-23,4 mJ/mm². Für die Medikation mit dem Placebo ergab sich eine Intensität von $21,177 \mathrm{~mJ} / \mathrm{mm}^{2}$ mit einer Streuung von 19,5-23,4 mJ/mm², und in der Kontrollmessung wurde eine Laserintensität von $21,32 \mathrm{~mJ} / \mathrm{mm}^{2}$ mit einer Streuung von 19,5-22,1$22,1 \mathrm{~mJ} / \mathrm{mm}^{2}$ gemessen. 


\subsection{Psychophysik}

Zunächst wurde mit allen Ergebnissen der subjektiven Schmerzwahrnehmung die Varianzanalyse, ANOVA, durchgeführt. Es wurden drei Haupteffekte unterschieden: KONDITION (Medikament oder Placebo), ZEIT (VOR; NACH 1-4), und die ZEIT x KONDITIONInteraktion. Hier zeigte sich im Vergleich zwischen den verschiedenen Messungen bezogen auf die KONDITION kein signifikanter Unterschied $(F(1,23)=2,38, \quad p=0,136)$. Die ZEIT-Komponente allerdings zeigte signifikante Unterschiede $(F(3,69)=10,89, \quad p<0,005)$. Die Interaktion zwischen ZEIT und KONDITION zeigte keine Signifikanz $(F(3,69)=0,50$; $p=0,680)$.

Die Probanden spürten also keinen signifikanten Unterschied zwischen den Messungen mit Placebo oder Medikament, die Schmerzstärke zu den verschiedenen Messzeitpunkten (VOR, NACH 1- $\mathrm{NACH}$ 4) wurde aber signifikant unterschiedlich, VOR stärker als $\mathrm{NACH}$, wahrgenommen. Der Vergleich zwischen der Kontrollgruppe und der Gruppe mit Medikation zeigte ebenfalls in der ZEIT-Komponente einen Unterschied $(F(3,39)=7,47 ; p<0,005)$, KONDITION $(F(2,26)=1,56 ; p=0,229)$ und ZEIT $x$ KONDITION $(F(6,78)=0,80 ; p=0,570)$ waren nicht signifikant.

Um alleine den Effekt der Stromstimulation auf die Schmerzwahrnehmung beurteilen zu können, führten wir eine univariante ANOVA durch. Unter den Bedingungen tDCS und Pergolid fand sich im Verlauf (also im Vergleich der Schmerzintensität vor der tDCS mit der Stärke der Schmerzwahrnehmung nach tDCS) eine signifikante Reduktion der Schmerzwahrnehmung $(F(4,92)=6,06, p<0,005)$.

Die post-hoc-Analyse zeigte eine signifikant niedrigere Schmerzwahrnehmung in den Messungen $\mathrm{NACH} 1-3$ als vor der Stimulation $(p<0,05)$. Unter der Placebo-Medikation fand sich im Verlauf eine signifikant niedrigere Schmerzwahrnehmung in der Messung NACH 2 als an allen anderen Zeitpunkten $(p<0,05)$. Der Faktor ZEIT zeigte signifikante Unterschiede $(F(4,92)=4,54, p=0,002)$. Im Kontrollexperiment 
ohne Medikation ergab sich in der univarianten ANOVA keine Signifikanz für den Faktor ZEIT $(F(4,52)=0,94), p=0,446)$.

\begin{tabular}{|c|c|c|c|c|c|c|}
\hline Kondition & Seite & vor & nach1 & nach2 & nach3 & nach4 \\
\hline \multirow{2}{*}{$\begin{array}{c}\text { Pergolid } \\
+ \\
\text { tDCS } \\
n=12\end{array}$} & links & $\begin{array}{c}4,91 \\
\pm \\
2,02\end{array}$ & $\begin{array}{c}4,37 \\
\pm \\
1,98\end{array}$ & $\begin{array}{c}4,06 \\
\pm \\
2,04\end{array}$ & $\begin{array}{c}4,25 \\
\pm \\
2,27\end{array}$ & $\begin{array}{c}4,89 \\
\pm \\
2,09\end{array}$ \\
\hline & rechts & $\begin{array}{c}5,05 \\
\pm \\
1,77\end{array}$ & $\begin{array}{c}4,30 \\
\pm \\
2,03\end{array}$ & $\begin{array}{c}3,66 \\
\pm \\
2,09\end{array}$ & $\begin{array}{c}4,50 \\
\pm \\
2.32\end{array}$ & $\begin{array}{c}4,65 \\
\pm \\
1,79\end{array}$ \\
\hline \multirow{2}{*}{$\begin{array}{c}\text { Plazebo } \\
+ \\
\text { tDCS } \\
n=12\end{array}$} & links & $\begin{array}{c}4,05 \\
\pm \\
1,34\end{array}$ & $\begin{array}{c}4,14 \\
\pm \\
1,41\end{array}$ & $\begin{array}{c}3,55 \\
\pm \\
1,37\end{array}$ & $\begin{array}{c}4,14 \\
\pm \\
1,72\end{array}$ & $\begin{array}{c}4,36 \\
\pm \\
1,48\end{array}$ \\
\hline & rechts & $\begin{array}{c}4,39 \\
\pm \\
1,39\end{array}$ & $\begin{array}{c}3,98 \\
\pm \\
1,66\end{array}$ & $\begin{array}{c}3,46 \\
\pm \\
1,34\end{array}$ & $\begin{array}{c}4,41 \\
\pm \\
1,91\end{array}$ & $\begin{array}{c}4,27 \\
\pm \\
1,32\end{array}$ \\
\hline \multirow{2}{*}{$\begin{array}{c}\text { Kontrolle } \\
n=7\end{array}$} & links & $\begin{array}{c}3,69 \\
\pm \\
1,76\end{array}$ & $\begin{array}{c}3,31 \\
\pm \\
1,56\end{array}$ & $\begin{array}{c}3,43 \\
\pm \\
2,15\end{array}$ & $\begin{array}{c}3,61 \\
\pm \\
1,79\end{array}$ & $\begin{array}{c}4,02 \\
\pm \\
1,40\end{array}$ \\
\hline & rechts & $\begin{array}{c}3,28 \\
\pm \\
1,15\end{array}$ & $\begin{array}{c}3,19 \\
\pm \\
1,32\end{array}$ & $\begin{array}{c}3,59 \\
\pm \\
1,88\end{array}$ & $\begin{array}{c}3,35 \\
\pm \\
1,90\end{array}$ & $\begin{array}{c}3,49 \\
\pm \\
1,55\end{array}$ \\
\hline
\end{tabular}

Tabelle 1: die Ergebnisse der NAS-Messungen nach rechter und linker Hand getrennt, alle fünf Messungen (gemittelte Werte aus jeweils 40 Laser-Stimulationen pro Messung)

Die Abbildung 4.1 zeigt die subjektiv bestimmte Schmerzintensität zu den verschiedenen Zeitpunkten (standardisierte Daten: die Ergebnisse NACH 1-4 wurden durch die Ergebnisse VOR geteilt.). Die Schmerzintensität unter Pergolid ist niedriger. Die Sternchen markieren signifikante Unterschiede zwischen den einzelnen Messungen im Zeitverlauf. 


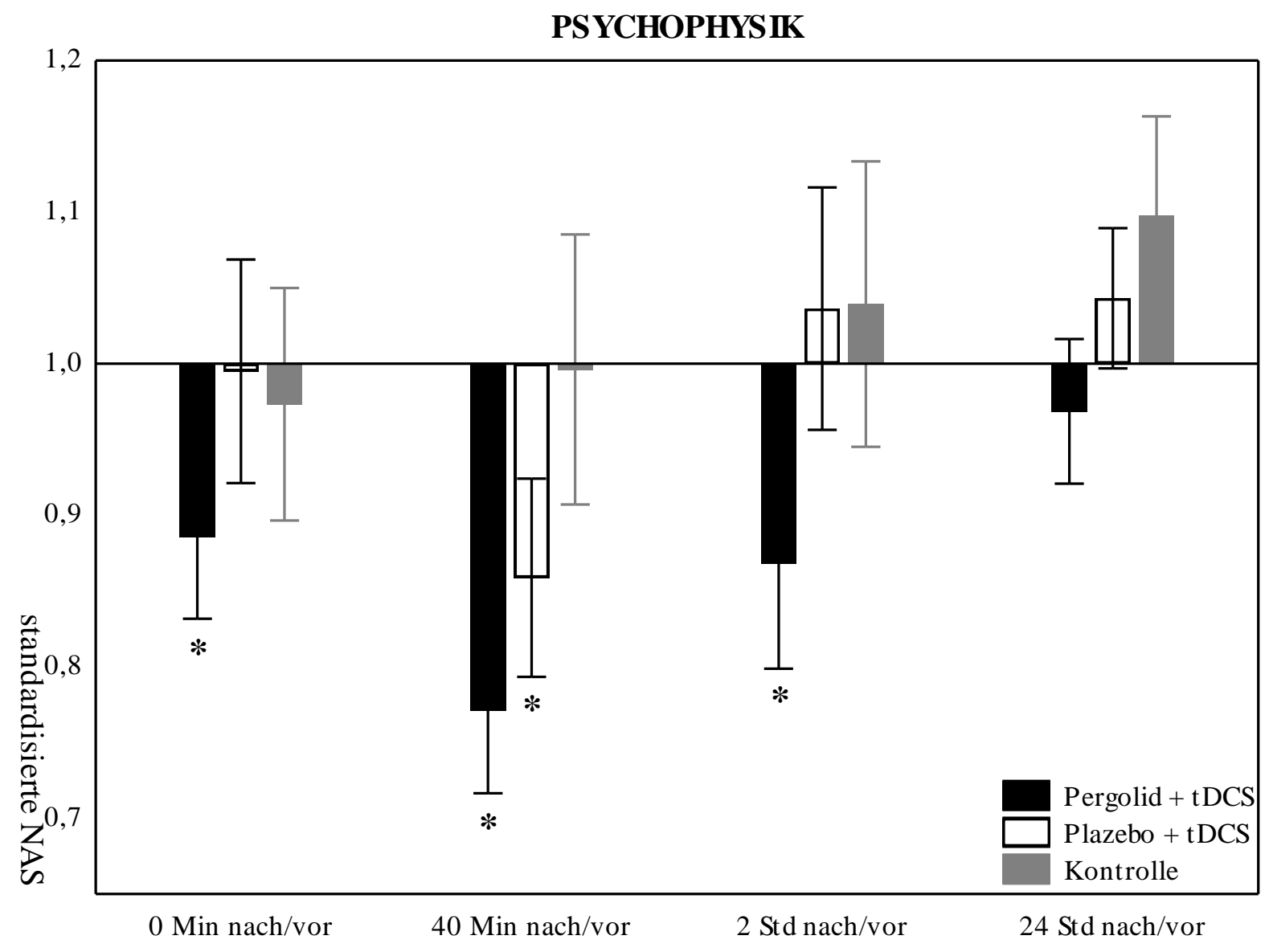

Abbildung 4-1: NAS der Schmerzwahrnehmung. Sternchen bedeuten, dass die zum entsprechenden Zeitpunkt angegebene Schmerzwahrnehmung signifikant niedriger war als die VOR der tDCS.

\section{$4.3 \quad$ Elektrophysiologie}

In allen EEGs konnten der N1-Komplex und der biphasische N2-P2Komplex eindeutig identifiziert werden. Aus allen aufgenommenen Sequenzen aus mindestens 40 Laserimpulsen wurden jeweils mindestens 35 eindeutig identifizierbare N2-P2 Komplexe ausgewählt. Messungen mit Artefakten wurden ausgeschlossen. 


\subsection{1 $\mathrm{N} 1$}

Für die erste negative Amplitude $\mathrm{N} 1$ gab es keine signifikanten Unterschiede über der Elektrode T3 in der ANOVA für KONDITION, ZEIT oder KONDITION x ZEIT (KONDITION: $F(1,23)=0,437, p=0,515$; ZEIT: $F(3,69)=0,14, p=0,937$; KONDITION x ZEIT: $F(3,69)=0,28, p=0,840)$. Auch für die T4 Elektrode ergab sich keine Signifikanz, weder für ZEIT $(F(3,69)=0,24, p=0,654)$, noch KONDITION $(F(1,23)=0,116, p=0,325)$, noch KONDITION x ZEIT $(F(3,69)=0,38, p=0,213)$.

Auch im Kontrollexperiment zeigten sich keine signifikanten Effekte von ZEIT, KONDITION, oder ZEIT x KONDITION ( $p>0,005)$.

\subsection{2 $\underline{\mathrm{N} 2}$}

Die KONDITION, also Medikament oder Placebo, ergab keine signifikanten Unterschiede, der Zeitpunkt und auch KONDITION $x$ ZEIT waren jedoch signifikant unterschiedlich. (KONDITION: $F(1,23)=0,12$, $p=0,737 ; \quad Z E I T: \quad F(3,69)=7,44, \quad p<0,005 ; \quad Z E I T \quad x \quad$ KONDITION: $F(3,69)=1,369 ; p=0,259)$. Betrachtet man nur die Elektrode $C z$, so zeigt sich ein signifikanter Unterschied zwischen den Zeitpunkten (ZEIT: $F(3,69)=5,65 ; p=0,001)$; zwischen Pergolid und Placebo allerdings gibt es auch für $\mathrm{Cz}$ keine signifikanten Unterschiede (KONDITION: $F(1,23)=0,91 ; p=0,349)$. Hier war die ZEIT $x$ KONDITION-Interaktion ebenfalls signifikant $(F(3,69)=3,67 ; p=0,016)$.

In der Post-hoc-Analyse konnten wir feststellen, dass die Amplitude unter Medikation mit Placebo nach 24 Stunden signifikant höher war als die Amplitude unter Pergolid $(p=0,006)$. Die Vergleiche der anderen Zeitpunkte zeigten allerdings keine signifikanten Unterschiede zwischen Placebo und Pergolid ( $p>0,05)$.

Die Einzelanalyse der Ableitung an $\mathrm{Pz}$ zeigte wieder signifikante Unterschiede zwischen den einzelnen Messzeitpunkten (ZEIT: $F(3,69)=6,82 ; p<0,005)$, KONDITION und ZEIT x KONDITION-Interaktion 
waren nicht signifikant (KONDITION: $F(1,23)=1,93, p=0,178$; ZEIT $x$ KONDITION: $F(3,69)=2,16, p=0,101)$.

Um die Ergebnisse der Kontrolluntersuchung ohne Medikation und tDCS mit den anderen beiden Untersuchungen zu vergleichen, wurden sie ebenfalls genormt und für alle Elektrodenpositionen in die ANOVA eingegeben. Auch hier war die ZEIT der signifikante Faktor $(p<0,05)$, KONDITION und KONDITION x ZEIT waren nicht signifikant $(p>0,05)$.

In der One-Way-ANOVA, die die Ergebnisse der einzelnen Konditionen untereinander prüft, fanden wir signifikante Unterschiede der verschiedenen Zeitpunkte unter Pergolid $(F(3,69)=3,65 ; p=0,017)$. Die Post-hoc-Analyse zeigte dann eine signifikante Verminderung der Amplitude im Vergleich zwischen der ersten (VOR) Messung und der zweiten Messung nach Stimulation (NACH 2) $(p=0,002)$.

Auch die Messungen mit Placebo zeigten in der One-Way-ANOVA Signifikanzen zwischen den Zeitpunkten (ZEIT: $F(3,69)=6,60 ; p<0,005)$. Signifikante Unterschiede zwischen den Messungen VOR und NACH 3 und NACH $1-2$ und NACH $3(p<0,05)$.

In der Kontrollgruppe zeigte die univariante ANOVA keinerlei signifikante Verminderungen der Amplituden zwischen den unterschiedlichen Zeitpunkten $(F(3,39)=1,57, p=0,211)$. 


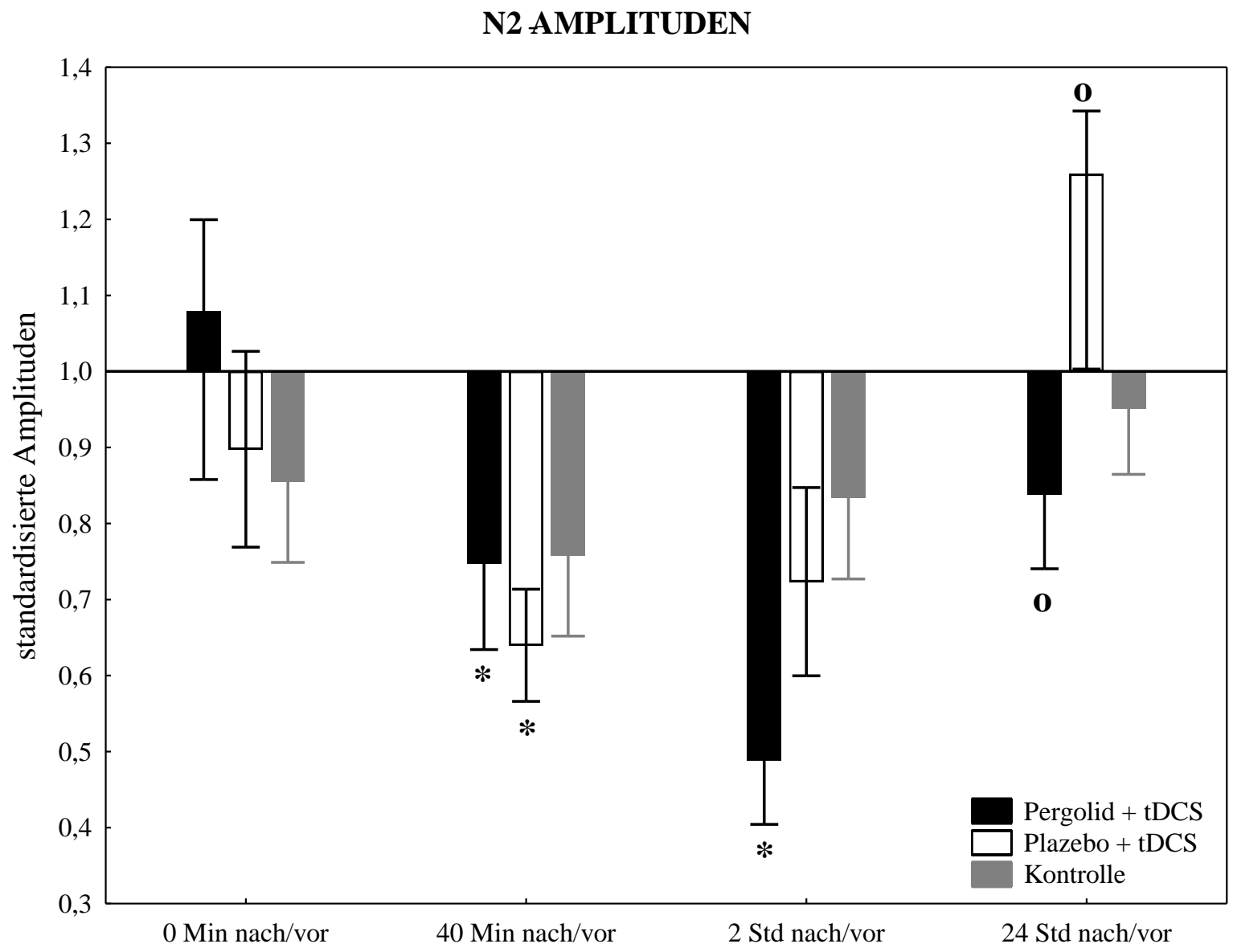

Abbildung 4-2: N2-Amplitudenhöhe: die signifikant unterschiedlichen

Messungen sind mit einem Stern versehen.

\subsection{3 $\underline{\mathrm{P} 2}$}

Auch für die zweite positive Amplitude P2 fanden wir in der ANOVA eine signifikante Veränderung der Amplitudenhöhe an den verschiedenen Messzeitpunkten (ZEIT: $p<0,05$ ). Zwischen den beiden Medikationen gab es keine signifikanten Unterschiede (KONDITION: p>0,05), auch die ZEIT x KONDITION-Interaktion ergab keine signifikanten Unterschiede (KONDITION x ZEIT: $p>0,05)$. Ebenso verhielt es sich im Vergleich 
zwischen den Ergebnissen der Kontrollgruppe und den Messungen unter Medikation: der Zeiteffekt war signifikant, KONDITION und ZEIT $x$ KONDITION waren nicht signifikant ( $p>0,05)$. 


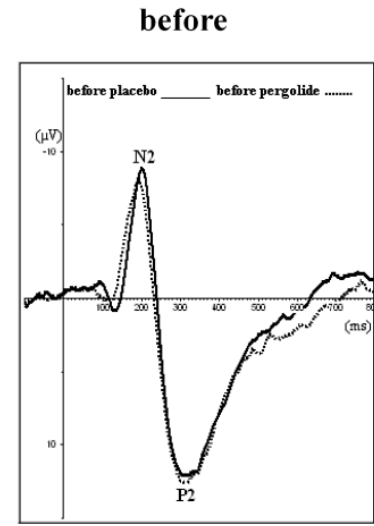

after $40 \mathrm{~min}$

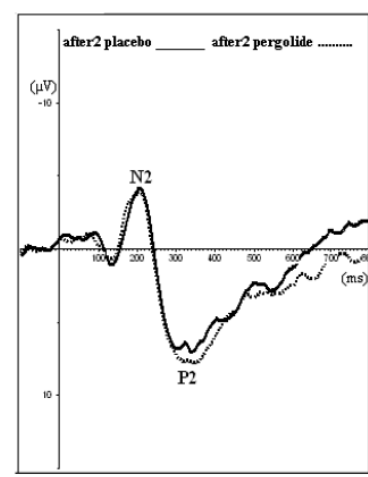

after 0 min

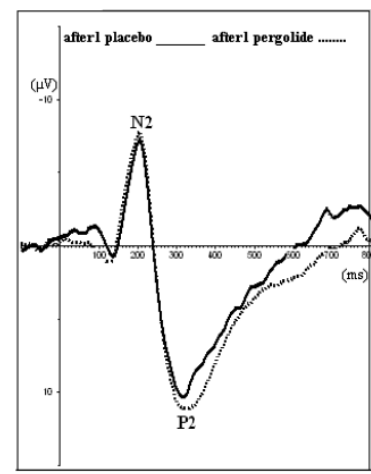

after 2 hours

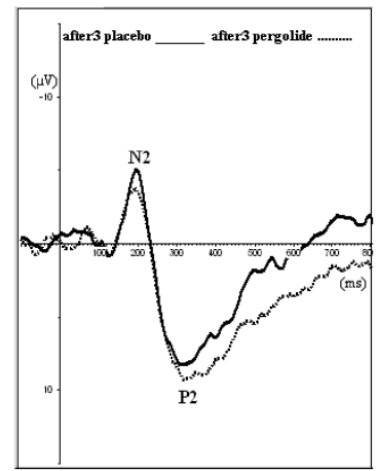

after 24 hours

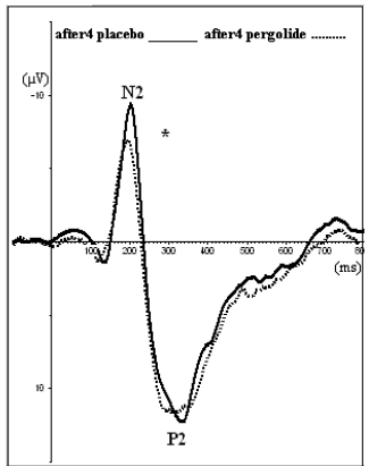

Abbildung 4-3: Mittelwert der LEPs beider Hände über Cz. Die dünne Linie zeigt die LEP unter Pergolid-Medikation, die dicke Linie unter Placebo. Das Sternchen zeigt signifikante Unterschiede zwischen den beiden Konditionen. 


\begin{tabular}{|c|c|c|c|c|c|c|}
\hline Kondition & Komponente & $\begin{array}{l}\text { vor } \\
\text { tDCS }\end{array}$ & $\begin{array}{c}\text { nach1 } \\
\text { tDCS } \\
\text { (0 Min) }\end{array}$ & $\begin{array}{c}\text { nach2 } \\
\text { tDCS } \\
\text { (40 Min) }\end{array}$ & $\begin{array}{c}\text { nach3 } \\
\text { tDCS } \\
\text { (2 Std) }\end{array}$ & $\begin{array}{c}\text { nach4 } \\
\text { tDCS } \\
\text { (24 Std) }\end{array}$ \\
\hline \multirow{2}{*}{$\begin{array}{c}\text { Pergolid } \\
+ \\
\text { tDCS } \\
n=12\end{array}$} & $\mathrm{~N} 2(\mu \mathrm{V})$ & $\begin{array}{c}-11,42 \\
\pm 6,40\end{array}$ & $\begin{array}{c}-10,09 \\
\pm 5,55\end{array}$ & $\begin{array}{r}-7,23 \\
\pm 4,49\end{array}$ & $\begin{array}{l}-7,32 \\
\pm 5,97\end{array}$ & $\begin{array}{l}-9,33 \\
\pm 5,33\end{array}$ \\
\hline & $\mathrm{P} 2(\mu \mathrm{V})$ & $\begin{array}{l}14,87 \\
\pm 5,54\end{array}$ & $\begin{array}{l}12,82 \\
\pm 6,12\end{array}$ & $\begin{array}{c}10,9 \\
\pm 6,14\end{array}$ & $\begin{array}{l}11,68 \\
\pm 7,58\end{array}$ & $\begin{array}{l}13,55 \\
\pm 5,90\end{array}$ \\
\hline \multirow{2}{*}{$\begin{array}{c}\text { Plazebo } \\
+ \\
\text { tDCS } \\
\mathrm{n}=12 \\
\end{array}$} & $\mathrm{~N} 2(\mu \mathrm{V})$ & $\begin{array}{c}-11,04 \\
\pm 5,82\end{array}$ & $\begin{array}{l}-9,36 \\
\pm 6,59\end{array}$ & $\begin{array}{l}-6,78 \\
\pm 4,23\end{array}$ & $\begin{array}{l}-7,38 \\
\pm 3,66\end{array}$ & $\begin{array}{c}-11,39 \\
\pm 5,32\end{array}$ \\
\hline & $\mathrm{P} 2(\mu \mathrm{V})$ & $\begin{array}{l}15,14 \\
\pm 6,96\end{array}$ & $\begin{array}{l}12,27 \\
\pm 7,11\end{array}$ & $\begin{array}{c}9,07 \\
\pm 5,44\end{array}$ & $\begin{array}{l}11,53 \\
\pm 5,23\end{array}$ & $\begin{array}{l}14,76 \\
\pm 5,49\end{array}$ \\
\hline \multirow{2}{*}{$\begin{array}{c}\text { Kontrolle } \\
n=7\end{array}$} & $\mathrm{~N} 2(\mu \mathrm{V})$ & $\begin{array}{l}-13,12 \\
\pm 7,52\end{array}$ & $\begin{array}{c}-11,83 \\
\pm 7,92\end{array}$ & $\begin{array}{l}-10,31 \\
\pm 6,44\end{array}$ & $\begin{array}{l}-8,67 \\
\pm 5,42\end{array}$ & $\begin{array}{c}-12,45 \\
\pm 7,75\end{array}$ \\
\hline & $\mathrm{P} 2(\mu \mathrm{V})$ & $\begin{array}{l}12,94 \\
\pm 3,51\end{array}$ & $\begin{array}{l}10,06 \\
\pm 3,21\end{array}$ & $\begin{array}{c}8,79 \\
\pm 5,25\end{array}$ & $\begin{array}{c}9,57 \\
\pm 3,83\end{array}$ & $\begin{array}{l}11,32 \\
\pm 2,05\end{array}$ \\
\hline
\end{tabular}

Tabelle 2: Durchschnittswerte und Standardabweichungen der LEPs aller Probanden über $\mathrm{Cz}$; rechte Hand

\begin{tabular}{|c|c|c|c|c|c|c|}
\hline Kondition & Komponent & $\begin{array}{l}\text { vor } \\
\text { tDCS }\end{array}$ & $\begin{array}{l}\text { nach1 } \\
\text { tDCS } \\
\text { (0 Min) }\end{array}$ & $\begin{array}{c}\text { nach2 } \\
\text { tDCS } \\
\text { (40 Min) }\end{array}$ & $\begin{array}{l}\text { nach3 } \\
\text { tDCS } \\
\text { (2 Std) }\end{array}$ & $\begin{array}{c}\text { nach4 } \\
\text { tDCS } \\
\text { (24 Std) }\end{array}$ \\
\hline \multirow{4}{*}{$\begin{array}{c}\text { Pergolid } \\
+ \\
\text { tDCS } \\
n=12\end{array}$} & \multirow{2}{*}{$\mathrm{N} 2(\mu \mathrm{V})$} & -11,73 & $-9,98$ & $-7,45$ & $-5,83$ & $-8,5$ \\
\hline & & $\pm 4,66$ & $\pm 5,52$ & $\pm 5,71$ & $\pm 7,01$ & $\pm 5,19$ \\
\hline & \multirow{2}{*}{$\mathrm{P} 2(\mu \mathrm{V})$} & 15,22 & 12,48 & 10,38 & 10,75 & 14,22 \\
\hline & & $\pm 6,27$ & $\pm 6,87$ & $\pm 6,40$ & $\pm 6,39$ & $\pm 7,03$ \\
\hline \multirow{4}{*}{$\begin{array}{c}\text { Plazebo } \\
+ \\
\text { tDCS } \\
n=12 \\
\end{array}$} & \multirow{2}{*}{$\mathrm{N} 2(\mu \mathrm{V})$} & $-11,57$ & $-9,16$ & $-5,56$ & $-6,37$ & $-11,52$ \\
\hline & & $\pm 6,96$ & $\pm 5,81$ & $\pm 4,15$ & $\pm 4,52$ & $\pm 6,30$ \\
\hline & \multirow{2}{*}{$\mathrm{P} 2(\mu \mathrm{V})$} & 15,55 & 11,83 & 9,41 & 11,61 & 15,58 \\
\hline & & $\pm 8,60$ & $\pm 6,61$ & $\pm 6,89$ & $\pm 8,02$ & $\pm 7,02$ \\
\hline \multirow{3}{*}{$\begin{array}{c}\text { Kontrolle } \\
n=7\end{array}$} & \multirow{2}{*}{$\mathrm{N} 2(\mu \mathrm{V})$} & $-13,22$ & $-10,68$ & $-8,48$ & $-9,15$ & $-11,89$ \\
\hline & & $\pm 6,70$ & $\pm 7,76$ & $\pm 4,95$ & $\pm 5,93$ & $\pm 5,84$ \\
\hline & $\mathrm{P} 2(\mu \mathrm{V})$ & $\begin{array}{r}11,95 \\
+352\end{array}$ & $\begin{array}{c}8,67 \\
+391\end{array}$ & $\begin{array}{r}9,46 \\
+385\end{array}$ & $\begin{array}{c}9,61 \\
+4,46\end{array}$ & 12,45 \\
\hline
\end{tabular}

Tabelle 3: : Durchschnittswerte und Standardabweichungen der LEPs aller Probanden über $\mathrm{Cz}$; linke Hand 


\section{Diskussion}

Wir konnten mit der vorliegenden Studie zeigen, dass eine fünfzehnminütige kathodale Stromstimulation über dem primären Motorkortex die subjektive Schmerzwahrnehmung und die N2Komponente der Laser-evozierten Potenziale signifikant senken kann. Durch die Einnahme des Dopaminagonisten Pergolid wird die Dauer dieser Wirkung signifikant verlängert. Die N2-Komponente der LEPs konnte durch tDCS und Pergolid bis zu 24 Stunden gesenkt werden.

Die subjektive Schmerzwahrnehmung, gemessen mit der Numerischen Analogskala, blieb unter Placebo und tDCS für bis zu 40 Minuten, unter tDCS und Pergolid für bis zu zwei Stunden unterhalb des Ausgangswertes.

Unsere Vermutung, dass die Wirkung der kathodalen tDCS durch Pergolid verlängert werden kann, bestätigte sich also. Wir gehen davon aus, dass dieses durch die weiter oben bereits beschriebene Verminderung der kortikalen Exzitabilität durch die kathodale tDCS und die dopaminerge Verfestigung der veränderten neuronalen Plastizität zu erklären ist.

Fregni et al. setzten tDCS in verschiedenen Experimenten zur Schmerzreduktion bei Patienten mit chronischen Schmerzen ein (Fregni et al. 2006 a, c). Sowohl Patienten mit Fibromyalgie als auch Patienten mit zentralem Schmerz nach Wirbelsäulenverletzungen spürten eine Minderung ihrer Schmerzen durch die Stimulation. Sie benutzten allerdings anodale Stimulation, dieser Unterschied $\mathrm{zu}$ unseren Ergebnissen mit kathodaler Stimulation könnte durch die Unterschiede chronischer und akuter Schmerzverarbeitung erklärt werden. Auch von Csifcsak et al. wurde kathodale Stimulation zur Minderung der LEPAmplituden angewandt.

Im folgenden Teil soll nun den Fragen nachgegangen werden

1. wieso die Stimulation des Motorkortex die Minderung von Schmerzwahrnehmung und Amplituden bewirken kann; 
2. wie die verschiedenen Komponenten der LEPs zu erklären sind und welcher Mechanismus zu ihrer Verminderung führen könnte;

3. ob sich die tDCS grundsätzlich als sichere Methode zur Schmerzbehandlung eignet und

4. was es, auch im Zusammenhang mit vorherigen Studien, mit der Medikamenteninteraktion auf sich hat.

\subsection{Stimulation des Motorkortex}

Seit Jahren ist bekannt, dass der Motorkortex an der zentralen Schmerzverarbeitung beteiligt ist. Lende et al. beobachteten bereits vor über 40 Jahren, dass die Resektion des Motorkortex Patienten mit chronischen Schmerzen Erleichterung verschaffte (Lende et al. 1971). Es folgten Beobachtungen, nach denen die Stromstimulation des primären Motorkortex Schmerzminderung hervorruft (Tsubokawa et al. 1991, 1993, Csifcsak et al. 2009, Lefaucheur et al. 2001). Der Motorkortex gehört zur so genannten Schmerzmatrix (Peyron et al. 2007) und ist laut verschiedener BOLD(blood oxygen level dependent)-Studien in die Schmerzwahrnehmung bzw. Schmerzreaktion eingebunden (Peyron et al. 2000, 2007, Derbyshire et al. 1999).

Die positive Wirkung der Motorkortexstimulation auf Schmerzen wurde von Tsubokawa et al. zunächst an Tierexperimenten, dann an Patienten untersucht: durch invasive Stimulation des Motorkortex konnten bis dahin therapierefraktäre Thalamusschmerzen behandelt werden (Tsubokawa et al. 1991). Als mögliche Erklärung für die Wirkung der Motorkortexstimulation gilt, dass Schmerz eine Hyperaktivität thalamischer Neurone (z. B. nach einer Läsion des Tractus spinothalamicus) auslöst. Man geht davon aus, dass die Motorkortexstimulation eine Minderung der Thalamusaktivität bewirken kann und dieses die Schmerzempfindung günstig beeinflusst (Schwenkreis et al. 2007). García-Larrea et al. beobachteten in einer PET-Studie, dass die Stimulation des Motorkortex zunächst via 
kortikothalamischer Bahnen eine Erhöhung des rCBF im ventrolateralen Thalamus bewirkt. Dieser beeinflusst dann sekundär Teile des medialen nozizeptiven Systems (wie medialen Thalamus, anteriores Cingulum, das periaquäduktale Grau im oberen Hirnstamm und die vordere Inselrinde), auch hier wurde ein erhöhter rCBF nach Motorkortexstimulation festgestellt (García-Larrea et al. 1999). Die Wirkung der Stimulation des Motorkortex wird somit zum einen auf eine Beeinflussung der affektivmotivationalen Schmerzkomponente über den medialen Thalamus und den vorderen Teil des Gyrus cinguli, zum anderen auf eine Beeinflussung der Schmerzverarbeitung auf spinaler Ebene durch eine verstärkte deszendierende Hemmung zurück geführt (Schwenkreis et al. 2007). Durch diese Studie von García-Larrea et al. wird die Verbindung zwischen Motorkortex und Thalamus in der Schmerzreduktion in den Vordergrund gestellt, und auch für unsere Studie gehen wir von diesem Wirkmechanismus aus.

Unsere Ergebnisse sind im Einklang mit dem oben bereits erwähnten Experiment von Csifcsak et al. (2009), in dem die Schmerzwahrnehmung ebenfalls durch kathodale tDCS des primären Motorkortex herabgesetzt werden konnte.

Unilaterale Motorkortexstimulation bewirkte in unserem Experiment eine Schmerzreduktion nach Laserstimulation sowohl der rechten als auch der linken Hand. Diverse PET-Studien haben bereits erwiesen, dass Schmerz bilateral verarbeitet wird (Peyron et al. 2000). Dass die Stimulation eines Motorkortex wie in unserem Experiment eine beidseitige Wirkung hervorruft, beobachteten auch Bäumer et al. in einer Studie mit repetitiver Magnetstimulation (Bäumer et al. 2003). Es wird vermutet, dass durch die multiple Involvierung des Motorkortex in die Schmerzverarbeitung die Stimulation einer Seite ausreichend ist. 


\section{$5.2 \quad \underline{N} 1, \mathrm{~N} 2, \mathrm{P} 2$}

Laser-evozierte Potenziale sind eine etablierte Methode zur Untersuchung sowohl zeitlicher als auch örtlicher Repräsentation der Schmerzwahrnehmung im Kortex (Treede et al. 2003, Spiegel et al. 2000).

Die erste Komponente eines durch einen Schmerzreiz hervorgerufenen Potenzials ist die Negativierung N1 (nach ca. 140-170 ms). Sie wird wahrscheinlich im fronto-parietalen Operculum im sekundären somatosensorischen Kortex generiert, da ihr Maximum mit den Elektroden T3 und T4 in diesem Bereich gemessen wird (Treede et al. 2003). N1 wurde durch die tDCS nicht in verändert. Vermutlich wurde dieser Teil des Kortex nicht direkt durch die tDCS erreicht, oder die Intensität der Stimulation war nicht ausreichend, um auch hier eine Veränderung hervorzurufen.

Nach N1 folgt der Komplex aus N2-P2, der durch die Elektroden der Mittellinie ( $\mathrm{Fz}, \mathrm{Pz}$ und $\mathrm{Cz}$ ) am deutlichsten abgeleitet werden kann. N2 wird bilateral in der operculoinsulären Region und im anterioren Gyrus cinguli generiert und kann 160-220 ms nach dem Reiz abgeleitet werden (Garcia-Larrea et al. 2003). Danach kann die Positivierung P2 abgeleitet werden, 330-360 ms nach dem Reiz, und zwar hauptsächlich aus dem anterioren Gyrus cinguli. Die Rolle des anterioren Gyrus cinguli in der Schmerzwahrnehmung ist noch nicht abschließend geklärt, sicher ist, dass er in der affektiven Verarbeitung des Schmerzreizes eine Rolle spielt, und Büchel et al. zeigten in einer fMRI- Studie, dass auch eine Korrelation der Aktivität des ACC mit der Stärke des Schmerzes besteht (Büchel et al. 2002). Eine PET-Studie zeigte, dass der regionale zerebrale Blutfluss im rechten ACC und Thalamus durch kathodale tDCS signifikant reduziert wird (Lang et al. 2005). Dass die N2-Amplitude durch eine direkte Wirkung der tDCS den anterioren Gyrus cinguli verändert wird, ist aufgrund seiner Lage unwahrscheinlich. Wir gehen davon aus, dass über die Verbindung zwischen dem primären Motorkortex und 
prämotorischen Arealen und dem ACC durch dessen Stimulation die Amplitude N2 gesenkt wird.

Eine Reduktion der Amplituden nach wiederholter Laserstimulation findet allerdings im Rahmen einer normalen Habituation auch ohne tDCS statt (Weiss et al. 1997). Um herauszufinden, ob die Reduktion durch tDCS verstärkt wird, führten wir die Kontrollexperimente ohne tDCS und Medikation. Unsere Annahme, dass die Amplituden unter tDCS und Medikation stärker und länger gesenkt würden, bestätigte sich: Zwar kam es in der Kontrollgruppe zu einem nicht-signifikanten Absinken der Amplituden von P2 und N2, unter tDCS und Medikation kam es aber wie oben bereits gezeigt zu einer stärkeren, signifikanten Reduktion der N2Amplitude. In der Psychophysik ergaben sich in der Kontrollgruppe keine signifikanten Unterschiede in der Schmerzwahrnehmung vor und nach Stimulation.

\subsection{Sicherheit und mögliche Anwendungsgebiete der Methode}

Die tDCS ist, wie bereits oben erwähnt, eine noninvasive Methode zur elektrischen Stimulation des Gehirns. Es handelt sich, soweit bisher bekannt, um eine nebenwirkungsarme Methode, sofern bei der Stimulation die Sicherheitsrichtlinien berücksichtigt werden (Agnew und Mc Creery 1987, Nitsche et al. 2003 c). Die Stromstärke, die wir verwendeten ( $1 \mathrm{~mA} / 35 \mathrm{~cm}^{2}$ ) entspricht den Empfehlungen, die Nitsche et al. 2003 veröffentlichten.

Im Gegensatz zum Beispiel zur TMS werden durch tDCS keine Aktionspotenziale ausgelöst, sondern das Ruhemembranpotenzial der Neurone wird durch den kontinuierlichen Stromfluss verändert. Die Elektroden zur Stimulation werden in mit $\mathrm{NaCl}$ oder Leitungswasser getränkten Schwämmen auf die Kopfhaut aufgelegt. Die Schwämme sollten weder $\mathrm{zu}$ feucht noch $\mathrm{zu}$ trocken sein, um zum einen eine Vergrößerung der Kontaktfläche zu verhindern und zum anderen den 
Hautwiderstand genügend herabzusetzen, um Verbrennungen zu vermeiden. Nach der Stromapplikation ist häufig eine Hautrötung unter den Elektroden zu beobachten, die aber vermutlich auf eine harmlose neurogene Vasodilatation zurückzuführen ist. Um eine eventuelle Überempfindlichkeit der Haut der Probanden auszuschließen, sollte im Erstgespräch auch dezidiert nach Hauterkrankungen und Überempfindlichkeiten gefragt werden (Nitsche et al. 2008).

Agnew et al. gehen davon aus, dass die Stromstärke zur Auslösung Motorisch-evozierter Potenziale von extrakranial zwar sehr wohl ausreichen würde, um durch Stimulation über einen längeren Zeitraum Schäden am Gehirn hervorzurufen. Durch den Haut- und Gewebewiderstand wird die Stromstärke allerdings weit genug abgeschwächt, um einen Schaden zu vermeiden. Dennoch sollte selbstverständlich bei allen Stimulationsarten darauf geachtet werden, die Elektroden (bzw. die Spule) nicht über offenen Fontanellen, Fissuren oder Defekten in der Schädeldecke zu platzieren (Agnew et al. 1987, Nitsche et al. 2008).

Da die tDCS im Gegensatz zur TMS keine gleichzeitige Aktivität großer Neuronenverbände bewirkt, ist das Risiko für epileptische Anfälle kleiner, bzw. soweit bisher bekannt nicht gegeben (Antal und Paulus 2007). Weitere Vorteile gegenüber der TMS sind die einfachere Anwendbarkeit und bessere Transportfähigkeit der Geräte, die Methode ist kostengünstiger und man erhofft sich von der tDCS einen länger anhaltenden Effekt (Fregni et al. 2007, Lefaucheur et al. 2008). Außerdem kann eine Placebo-Stimulation gut durchgeführt werden, so dass eine bessere Möglichkeit zur Kontrolle der klinischen Effekte besteht (Fregni et al. 2006 a).

Für die Sicherheit der Methode spricht auch eine große Studie, die die Nebenwirkungen der tDCS per Fragebogen an über 100 Probanden untersuchte. Ernste Nebenwirkungen wurden nicht beobachtet, nur 11\% der Probanden verspürten leichte Kopfschmerzen nach der Stimulation, außerdem wurde über Kribbeln und Jucken während der Stimulation 
berichtet. Über Übelkeit berichteten nur 2,9\% der Befragten, insgesamt wurde die Prozedur nur von wenigen als leicht unangenehm bewertet, und die Mehrheit der Probanden bemerkte keinen Unterschied zwischen anodaler, kathodaler oder Placebostimulation (Poreisz et al. 2007).

Es werden noch andere Ansätze verfolgt, die tDCS klinisch und experimentell zu nutzen: ein leicht positiver Effekt auf die motorischen Fähigkeiten von Patienten mit durch Schlaganfall bedingten Paresen konnte nachgewiesen werden (Fregni et al. 2005, Hummel et al. 2005). Ein positiver Effekt und kurzdauernde Besserung für Tinnituspatienten wurden von Fregni et al. gezeigt (Fregni et al. 2006 b). Speziell in der Schmerzbehandlung fand man eine Verbesserung der Symptome durch tDCS. Von Fregni et al. wurde sie zur Behandlung von Fibromyalgie und Patienten mit therapierefraktärem Schmerz nach Rückenmarksschäden eingesetzt. Sie erzielten in beiden Fällen durch die Stimulation des Motorkortex gute Ergebnisse (Fregni et al. 2006 a, c).

Des Weiteren hat die tDCS Auswirkungen auf motorische und visuelle Hirnfunktionen. Nitsche et al. zeigten, dass sich die Lernleistung bei motorischem Lernen durch tDCS verbessern lässt (Nitsche et al. 2003 a). Die visuelle Diskrimminationsleistung kann durch kathodale Stimulation über dem visuellen Kortex verbessert werden (Antal et al. 2004).

\subsection{Verlängerung der Wirkung der tDCS durch Pergolid}

Wir benutzten den Dopaminrezeptor-Agonist Pergolid, um eine Verlängerung der antinozizeptiven Wirkung der kathodalen tDCS zu erreichen und konnten nachweisen, dass im Vergleich zur reinen tDCS zu der mit Pergolid-Applikation tatsächlich eine Wirkungsverlängerung bis zu 24 Stunden erreicht werden kann.

Der Einfluss des Dopaminrezeptors auf die durch die tDCS vermittelte Neuroplastizität wurde in mehreren Studien beschrieben. Die 
erwünschten Nachwirkungen der tDCS sind NMDA-Rezeptor abhängig (Liebetanz et al. 2002, Nitsche et al. 2003 b), ersichtlich daraus, dass die Einnahme des NMDA-Rezeptor-Blockers Dextromethorphan sowohl die Nachwirkungen anodaler als auch kathodaler Stimulation verhindert. Wie bereits oben beschrieben, sind die lang anhaltenden NMDA-Rezeptorabhängigen Veränderungen der kortikalen Erregbarkeit ein Mechanismus, der über LTP und LTD zur Neuroplastizität beisteuert (Lang et al. 2005). Tierexperimente haben gezeigt, dass diese Effekte durch dopaminerge Mechanismen unterstützt werden (Otani et al. 1998, Bailey et al. 2000, Huda et al. 2001). Durch die Beeinflussung der cAMPund $\mathrm{Ca}^{2+}$-Spiegel nimmt Dopamin Einfluss auf LTP und LTD im Hippocampus, Striatum und im präfrontalen Cortex (Frey et al. 1993, Jay et al. 1998, Gurden et al. 2000, Spenser und Murphy 2002).

Wir gehen davon aus, dass über das von den Probanden eingenommene Pergolid die NMDA-Rezeptor-abhängige und tDCS-vermittelte Veränderung der neuronalen Erregbarkeit stabilisiert und verlängert werden konnte. Unsere Ergebnisse unterstützen und erweitern die Beobachtungen von Nitsche et al. (2006), die zeigten, dass die Wirkung der tDCS generell durch D2-Rezeptor-Stimulation durch Pergolid verlängert werden kann.

Von einer ursächlichen antinozizeptiven Wirkung des Pergolids selbst ist dabei nicht auszugehen, da zum einen nur eine sehr geringe Einmaldosis von $0,025 \mathrm{mg}$ verabreicht wurde und zum anderen bisher nichts über eine solche Wirkung des Medikaments bekannt ist.

Um mehr über die Mechanismen der tDCS zu erfahren und eventuell auf die Beteiligung weiterer Rezeptoren schließen zu können, sind in mehreren Studien verschiedene Medikamente mit tDCS kombiniert worden. NMDA-Rezeptoren wird eine zentrale Rolle für die Entstehung von Nacheffekten der tDCS zugeschrieben (Liebetanz et al. 2002, Nitsche et al. 2004). Dieser Effekt wird wiederum dopaminerg unterstützt (Nitsche et al. 2006), so dass wir durch eine verstärkte Aktivierung des 
Dopaminrezeptors durch Pergolid dessen unterstützenden Effekt auf die NMDA-Rezeptor-vermittelte Neuroplastizität verlängern konnten. 


\section{$6 \quad$ Zusammenfassung und Ausblick}

Unsere Studie zeigt, dass die schmerzmindernde Wirkung der transkraniellen kathodalen Gleichstromstimulation durch den Dopaminagonisten Pergolid verlängert werden kann. Sowohl die subjektive Schmerzwahrnehmung, gemessen anhand der Numerischen Annalogskala, als auch die Amplituden der Laser-evozierten Potenziale konnten mit Pergolid signifikant länger als unter reiner tDCS gesenkt werden.

Im Hinblick auf eine mögliche therapeutische Anwendung der tDCS zur Behandlung von Schmerzen könnte diese effektiver und in größeren Zeitabständen realisiert werden, was in der Anwendung im klinischen Alltag einen Vorteil für die Patienten und die Behandelnden darstellen würde. 


\section{$7 \quad$ Literatur}

Agnew W, McCreery D (1987): Considerations for safety in the use of extracranial stimulation for motor evoked potentials. Neurosurgery $\underline{20}, 143-147$

Antal A, Paulus W (2007): Transkranielle Gleichstromstimulation: Neues Werkzeug in der Schmerztherapie? Akt Neurol 34, 530-533

Antal A, Nitsche MA, Kruse W, Kincses T, Hoffmann K, Paulus W (2004): Direct current stimulation over V5 enhances visuomotor coordination by improving motion perception in humans. J Cogn Neurosci 16, 521-527

Bailey C, Giustetto M, Huang Y, Hawkins R, Kandel R (2000):Is heterosynaptic modulation essential for stabilizing Hebbian plasticity and memory? Nat Rev Neurosci $1,11-20$

Basbaum A, Jessel T: The Perception of Pain; in: Principles of Neural Science, 4. Auflage; hrsg. V. Kandel E, Schwartz J, Jessel T; Mc Graw Hill-Verlag o. O., USA 2000, 472-491

Baudewig J, Nitsche M, Paulus W, Frahm J (2001): Regional modulation of BOLD MRI responses to human sensorimotor activation by transcranial direct current stimulation. Magn Reson Med 45, 196-201

Bäumer T, Lange R, Liepert J, Weiller C, Siebner H, Rothwell J, Münchau A (2003): Repeated premotor rTMS leads to cumulative plastic changes of motor cortex excitability in humans. Neuroimage $\underline{20}, 550-560$

Bindman L, Lippold O, Redfearn J (1964): The action of brief polarizing currents on the cerebral cortex of the rat (1) during current flow and (2) in the production of long-lasting after-effects. J Physiol 172, 369-382 
Birbaumer N, Schmidt R: Lernen und Gedächtnis; in: Physiologie des Menschen, 30. Auflage; hrsg. v. Schmidt R und Lang F; Springer-Verlag Heidelberg 2007, 223-238

Bromm B, Treede $\mathrm{R}$ (1983): $\mathrm{Co}_{2}$ laser radiant heat pulses activate C nociceptors in man. Pflügers Arch $\underline{399}, 155-156$

Brown J (2001): Motor cortex stimulation. Neurosurg Focus 11, Article 5

Büchel C, Bornhovd K, Quante M, Glauche V, Bromm B, Weiller C (2002): Dissociable neural responses related to pain intensity, stimulus intensity, and stimulus awareness within the anterior cingulate cortex: a parametric single-trial laser functional magnetic resonance imaging study. J Neurosci $\underline{22}$, 970-976

Cooke S, Bliss T (2006): Plasticity in the human central nervous system. Brain $\underline{129}, 1659-1673$

Creach C, Henry P, Caille J, Allard M (2000): Functional MR imaging analysis of pain-related brain activation after acute mechanical stimulation. Am $\mathrm{J}$ Neuroradiol 21, 1402-1406

Creutzfeld O, Fromm G, Kapp H (1962): influence of transcortical d-c currents on cortical neuronal activity. Exp Neurol $\underline{5}, 436-452$

Cruccu G, Anand P, Attal N, Garcia-LarreaL, Haanpää M, Jørum E, Serra J, Jensen TS (2004): EFNS guidelines on neuropathic pain assessment. Eur J Neurol 11(3), 153-162

Csifcsak G, Antal A, Hillers F, Levold M, Bachmann C, Happe S, Nitsche MA, Ellrich J, Paulus W (2009): Modulatory effect of transcranial direct current stimulation on Laser evoked potentials. Pain Med 10, 122-132 
Deleu D, Northway M, Hanssens Y (2002): Clinical pharmacokinetic and pharmacodynamic properties of drugs used in the treatment of Parkinson's disease. J Pharmacokinet $\underline{4}, 261-309$

Derbyshire SW (1999): Meta-Analysis of Thirty-Four Independent Samples Studied Using PET Reveals a Significantly Attenuated Central Response to Noxious Stimulation in Clinical Pain Patients. Curr Rev Pain 3: 265-280.

Fregni F, Boggio P, Mansur C, Wagner T, Ferreira M, Lima M, Rigonatti S, Marcolin M, Freedman S, Nitsche M, Pascual-Leone A (2005): Transcranial direct current stimulation of the unaffected hemisphere in stroke patients. Neuroreport $\underline{16}, 1551-1555$

Fregni $F$, Boggio $P$, Lima M, Ferraira M, Wagner T, Rigonatti S, Castro A, Souza D, Riberto M, Freedman S, Nitsche M, Pascual-Leone A (2006 a): A sham-controlled, phase II trial of transcranial direct current stimulation for the treatment of central pain in traumatic spinal cord injury. Pain 122, 197-209

Fregni F, Marcondes R, Boggio P, Marcolin M, Rigonatti S, Sanchez T, Nitsche M, Pascual-Leone A (2006 b): Transient tinnitus suppression induced by repetitive transcranial magnetic stimulation and transcranial direct current stimulation. Eur J Neurol 13, 996-1001

Fregni F, Gimenes R, Valle A, Ferreira M, Rocha R, Natalle L, Bravo R, Rigonatti S, Freedman S, Nitsche M, Pascual-Leone A, Boggio P (2006 c): A randomized, sham-controlled, proof of principle study of transcranial direct current stimulation for the treatment of pain in fibromyalgia. Arthritis Rheum $\underline{54}$, 3988-3998 
Fregni F, Freedman S, Pascual-Leone A (2007): Recent advances in the treatment of chronic pain with non-invasive brain stimulation techniques. Lancet Neurol $\underline{6}, 188-191$

Frey U, Huang Y, Kandel E (1993): Effects of cAMP stimulate a late stage of LTP in hippocampal CA1 Neurons. Science 260, 1661-1664

Frot M, Mauguière F (2003): Dual representation of pain in the operculo-insular cortex in humans. Brain $\underline{126}, 438-450$

García-Larrea L, Peyron R, Mertens P, Gregoire M, Lavenne F, Le Bars D, Convers P, Mauguière F, Sindou M, Laurent B (1999): Electrical stimulation of motor cortex for pain control: a combined PET-scan and electrophysiological study. Pain $\underline{83}, 259-273$

García-Larrea L, Frot M, Valeriani M (2003): Brain generators of laser-evoked potentials: from dipoles to functional significance. Neurophysiol Clin $\underline{33}, 279-$ 292

Gurden H, Takita M, Jay T (2000): Essential role of D1 but not D2 receptors in the NMDA receptor-dependent long-term-potentiation at hippocampal-prefrontal cortex synapses in vivo. J Neurosci $\underline{20}, \mathrm{RC} 106$

Heckmann M, Dudel J: Erregungsleitung und synaptische Übertragung; in: Physiologie des Menschen, 30. Auflage; hrsg. v.Schmidt R, Lang F; Springer Verlag Heidelberg 2007, 78-110

Hirayama A, Saitoh Y, Kishima H, Shimokawa T, Oshino S, Hirata M, Kato A, Yoshimine $T$ (2006): Reduction of intractable deafferntation pain by navigationguided repetitive transcranial magnetic stimulation of the primary motor cortex. Pain 122, 22-27 
Huda K, Salunga T, Matsunami K (2001): Dopaminergic inhibition of excitatory inputs onto pyramidal tract neurons in cat motor cortex. Neurosci Lett $\underline{307}, 175-$ 178

Hummel F, Celnik P, Giraux P, Floel A, Wu W, Gerloff C, Cohen L (2005): Effects of non-invasive cortical stimulation on skilled motor function in chronic stroke. Brain $\underline{128}, 490-499$

Jay T (2003): Dopamine: a potential substrate for synaptic plasticity and memory mechanisms. Prog Neurobiol 69(6), 375-390

Jay T, Gurden H, Yamaguchi T (1998): Rapid increase in PKA activity during long-term potentiation in the hippocampal afferent fiber system to the prefrontal cortex in vivo. Eur J Neurosci $\underline{10}, 3302-3306$

Lang N, Siebner H, Ward N, Lee L, Nitsche M, Paulus W, Rothwell J, Lemon R, Frackowiak R (2005): How does transcranial DC stimulation of the primary motor cortex alter regional neuronal activity in the human brain? Eur J Neurosci $\underline{22}, 495-504$

Lefaucheur JP, Drouot X,Nguyen JP (2001): Interventional neurophysiology for pain control: duration of pain relief following repetitive transcranial magnetic stimulation of the motor cortex. Neurophysiol Clin $\underline{31}, 247-252$

Lefaucheur JP, Antal A, Ahdab R, Ciampi de Andrade D, Fregni F, Khedr E, Nitsche M, Paulus W (2008): The use of repetitive transcranial magnetic stimulation (rTMS) and transcranial direct current stimulation (tDCS) to relieve pain. Brain Stim 1, 337-344

Lende R, Kirsch W, Druckman R (1971): Relief of facial pain after combined removal of precentral and postcentral cortex. J Neurosurg $\underline{34(4)}, 537-543$ 
Liebetanz D, Nitsche MA, Tergau F, Paulus W (2002): Pharmacological approach tot he mechanisms of transcranial dc-stimulation-induced after-effects of human motor cortex excitability. Brain $\underline{125}, 2238-2247$

Lippold O, Redfearn J (1964): Mental Changes Resulting from the Passage of Small Direct Currents Through the Human Brain. Br J Psychiatry $\underline{110}$, 768-772

Lomo T (1966): Frequency potentiation of excitatory synaptic activity in the dentate area oft he hippocampal formation. Acta Physiol Scand $\underline{68}, 128$

Lomo T (2003): The discovery of long-term potentiation. Philos Trans R Soc Lond B Biol Sci $\underline{358}, 617-620$

Mouraux A, Plahgki L (2004): Single-trial detection of human brain responses evoked by laser activation of A-delta-nociceptors using the wavelet transform of EEG epochs. Neurosci Lett 361(1-3), 241-244

Mouraux A, Guérit JM, Plahgki L (2003): Non-phase locked electroencephalogram (EEG) responses to $\mathrm{CO}_{2}$ laser skin stimulationsmay reflect central interactions between A-delta- and C-fibre afferent volleys. Clin Neurophysiol $114,710-722$

Nitsche MA, Paulus W (2000): Excitability changes induced in the human motor cortex by weak transcranial direct current stimulation. J Physiol $\underline{527}$, 633-639

Nitsche MA, Paulus W (2001): Sustained excitability elevations induced by transcranial DC motor cortex stimulation in humans. Neurology $\underline{57}, 1899-1901$

Nitsche MA, Schauenburg A, Lang N, Liebetanz D, Exner C, Paulus W, Tergau F (2003 a): Facilitation of implicit motor learning by weak transcranial direct current stimulation of the primary motor cortex in the human. $\mathrm{J}$ Cogn Neurosci $\underline{15}, 619-626$ 
Nitsche MA, Fricke K, Henschke U, Schlitterlau A, Liebetanz D, Lang N, Henning S, Tergau F, Paulus W (2003 b): Pharmacological modulation of cortical excitability shifts induced by transcranial direct current stimulation in humans. J Physiol $\underline{533}$, 293-301

Nitsche MA, Liebetanz D, Lang N, Antal A, Tergau F, Paulus W (2003 c): Safety criteria for transcranial direct current stimulation (tDCS) in humans. Clin Neurophysiol $114(11), 2220-2222$

Nitsche MA, Grundey J, Liebetanz D, Lang N, Tergau F, Paulus W (2004): Catecholaminergic consolidation of motor cortical neuroplasticity in humans. Cereb Cort $\underline{14}, 1240-1245$

Nitsche MA, Lampe C, Antal A, Liebetanz D, Lang N, Tergau F, Paulus W (2006): Dopaminergic modulation of long-lasting direct current-induced cortical excitability changes in the human motor cortex. Eur J Neurosci $\underline{23}, 1651-1657$

Nitsche MA, Leonardo G, Wassermann E, Priori A, Lang N, Antal A, Paulus W, Hummel F, Boggio P, Fregni F, Pascual-Leone A (2008): Transcranial direct current stimulation: State of the art 2008. Brain Stim 1, 206-23

Otani S, Blond O, Desce JM, Crépel F (1998): Dopamine facilitates long-term depression of glutamatergic transmission in rat prefrontal cortex. Neuroscience $\underline{85(3)}, 669-676$

Pape HC: Integrative Funktionen des Gehirns; in: Physiologie, 5. Auflage; hrsg. v. Klinke R, Pape HC, Silbernagl S; Georg-Thieme Verlag, Stuttgart. 2005, 801831

Paulus W (2004): Outlasting Excitability Shifts Induced by Direct Current Stimulation of the Human Brain. Suppl Clin Neurophysiol $\underline{57}$, 708-714 
Peyron R, Laurent B, García-Larrea L (2000): Functional imaging of brain responses to pain. A review and meta-analysis. Neurophysiol Clin 30, 263-288

Peyron R, Kupers R, Jehl JL, García-Larrea L, Convers P, Barral FG, Laurent B (2007): Central representation of the RIII flexion reflex associated with overt motor reaction: an fMRI study. Neurophysiol Clin 37, 249-259

Plaghki L, Mouraux A (2005): EEG and laser stimulation as tools for pain research. Curr Opin Investig Drugs $\underline{6(1)}, 58-64$

Ploner M, Schnitzler A (2004): Kortikale Repräsentation von Schmerz. Nervenarzt $\underline{75}, 962-969$

Ploner M, Gross J, Timmermann L, Schnitzler A (2002): Cortical representation of first and second pain sensation in humans. Proc Natl Acad Sci USA 99(19), 12444-12448

Poreisz C, Boros K, Antal A, Paulus W (2007): Safety aspects of transcranial direct current stimulation concerning healthy subjects and patients. Brain Res Bull $\underline{72}, 208-214$

Priori A (2003): Brain polarization in humans: areappraisal of an old tool for prolonged non-invasive modulation of brain excitability. Clin Neurophysiol $\underline{114}$, 589-595

Purpura D, McMurty J (1964): Intracellular activities and evoked potential changes during polarization of motor cortex. J Neurophysiol $\underline{28}, 166-185$

Schaible H-G, Schmidt R: Nozizeption und Schmerz; in: Physiologie des Menschen, 30. Auflage; hrsg. v. Schmidt R und Lang F; Springer-Verlag Heidelberg 2007, 325-342 
Schwenkreis P, Pleger B, Tegenthoff M: Schmerz; in: Das TMS-Buch Transkranielle Magnetstimulation, 1. Auflage; hrsg. v. Siebner H, Ziemann U; Springer-Verlag, Berlin 2007, 599-607

Sheffield L, Mowbray R (1968): The effects of polarization on normal subjects. Br J Psychiatry 114, 225-232

Spenser J, Murphy K (2002): Activation of cyclic AMP-dependent protein kinase is required for long-term enhancement at corticostriatal synapses in rats. Neurosci Lett $\underline{329}$, 217-221

Spiegel J, Hansen C, Treede R (2000): Clinical evaluation criteria for the assessment of impaired pain sensitivity by thulium-laser evoked potentials. Clin Neurophysiol 111, 725-735

Timmermann L, Ploner M, Haucke K, Schmitz F, Baltissen R, Schnitzler A (2001): Differential Coding of Pain Intensity in the Human Primary and Secondary Somatosensory Cortex. J Neurophysiol 투, 1499-1503

Treede R, Lorenz J, Baumgärtner U (2003): Clinical usefulness of laser-evoked potentials. Neurophysiol Clin $\underline{33}, 303-314$

Tsubokawa T, Katayama Y, Yamamoto T, Hirayama T, Koyama S (1991): Treatment of thalamic pain by chronic motor cortex stimulation. Pacing Clin Electrophysiol 14, 131-134

Tsubokawa T, Katayama Y, Takamitsu Y, Hirayama T, Koyama S (1993): Chronic motor cortex stimulation in patients with thalamic pain. J Neurosurg $\underline{78}$, 393-401 
Weiss T, Kumpf K, Ehrhardt J, Gutberlet I, Miltner W (1997): A bioadaptive approach for experimental pain research in humans using laser-evoked brain potentials. Neurosci Lett 227, 95-98

Ziemann U, Bruns D, Paulus W (1996): Enhancement of human motor cortex inhibition by the dopamine receptor agonist pergolide: evidence from transcranial magnetic stimulation. Neurosci Lett $\underline{208}, 187-190$

Ziemann U, Tergau F, Bruns D, Baudewig J, Paulus W (1997): Changes in human motor cortex excitability induced by dopaminergic and anti-dopaminergic drugs. Electroencephalogr Clin Neurophysiol 105, 430-437 


\section{$8 \quad$ Tabellen- und Abbildungsverzeichnis}

Abbildung 2-1:

Abbildung 2-2:

Abbildung 2-3:

Abbildung 2-4:

Abbildung 3-1:

Abbildung 4-1:

Abbildung 4-2:

Abbildung 4-3:

Tabelle 1:

Tabelle 2:

Tabelle 3:
Seite 16

Seite 19

Seite 22

Seite 25

Seite 33

Seite 37

Seite 40

Seite 42

Seite 36

Seite 43

Seite 43 


\section{$9 \quad$ Anhang: Teilnehmerinformationen}

Der folgende Aufklärungsbogen wurde den Probanden zusammen mit der Einverständniserklärung ausgehändigt.

Zentrum Neurologie

Abteilung Neurologie (Prof. Dr. Bähr)

Abteilung Klinische Neurophysiologie (Prof. Dr. W. Paulus)

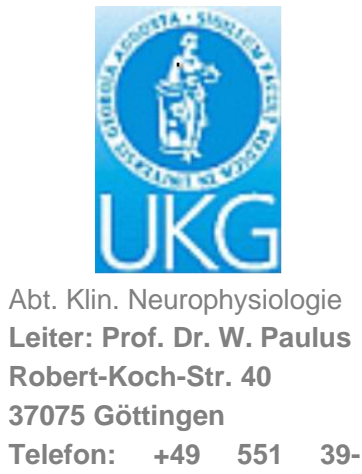

Telefax: +49 $55139-8126$

\section{Information für Teilnehmerinnen und Teilnehmer über die Untersuchung:}

\section{Einfluss schwachen transkraniell applizierten Gleichstroms auf Schmerzschwellen und sensorische Diskriminationsschwellen}

Sehr geehrte Teilnehmerin, sehr geehrter Teilnehmer!

Wir möchten Sie bitten, an o.g. wissenschaftlicher Untersuchung teilzunehmen und möchten Ihnen den Ablauf der Studie anhand dieses Informationsbogens erläutern. Sie selbst werden nicht unmittelbar von dieser Studie profitieren, die Ergebnisse ermöglichen es uns jedoch, weiterführende Erkenntnisse über Ursachen und Therapiemöglichkeiten von Schmerz zu gewinnen.

Es soll überprüft werden, ob sich Schmerzschwellen, wahrgenommene Schmerzintensität sowie die Unterscheidungsschwellen für verschiedene sensorische Modalitäten durch die Applikation schwachen Gleichstroms über die Kopfhaut verändern. Um die Wahrnehmungs-Intensität eines sensiblen Reizes zu bestimmen, werden wir die Schmerzschwelle und die Schmerzintensität messen. Dafür werden kurze Hitzeimpulse berührungslos durch einen Lichtleiter (einen sog. Laser) auf das zu untersuchende Hautareal an der Hand appliziert. Diese Reize erzeugen einen kurzzeitigen stechenden Schmerz. Hierdurch entstehen im Gehirn bestimmte Potentialmuster, die über eine Hirnstromkurve (EEG) schmerzlos gemessen und anschließend analysiert werden können. $\mathrm{Zu}$ Verletzungen oder dauerhaften Schädigungen kommt es hierbei nicht. Außerdem werden wir die von Ihnen 
wahrgenommenen Unterscheidungsschwellen für verschiedene sensible Modalitäten (Vibration, Druckschmerz, mechanischer Schmerz, Nadelreizstimulation) untersuchen.

In den Sitzungen werden zusätzlich zu den o.g. EEG-Elektroden zwei Elektroden auf Ihrer Kopfhaut angebracht. Über diese Elektroden fließt während der Untersuchung für 15 Minuten ein schwacher Gleichstrom. Dieser Strom ist für Sie nicht oder allenfalls sehr geringfügig wahrnehmbar. Dieses nicht invasive (=nicht verletzende) und schmerzfreie Verfahren wurde bereits in mehreren Untersuchungen angewendet und hat sich als risiko- und nebenwirkungsarm erwiesen. Selten und lediglich nach kontinuierlicher Gleichstromapplikation über Stunden traten leichte Kopfschmerzen und Hautreizungen im Bereich der Elektroden auf, die sich relativ schnell vollständig zurückbildeten.

In den allen Sitzungen ist darüber hinaus die Anwendung von Medikamenten geplant, um deren Einfluss auf die gemessenen Parameter zu bestimmen. Angewendet wird jeweils eines der Medikamente Pergolid, D-Cycloserin oder ein wirkstofffreies Medikament, ein sog. Placebo, jeweils als Tablette oder Kapsel. Alle diese Medikamente sind in ihren Wirkungen und Nebenwirkungen gut bekannt. Die Einnahme soll jeweils kurz vor Beginn der Untersuchungen erfolgen. Wir weisen darauf hin, dass die Führen eines Kraftfahrzeuges oder die Arbeiten an selbst- bzw. fremdgefährdenden Maschinen nach Einnahme der Medikamente bis zum Morgen des Tages nach der Untersuchung unterbleiben muss, da eine deutliche Beeinträchtigung der Fahrtüchtigkeit vorliegen kann. 30 min vor der Gabe der Pergolid-Medikation werden zur Verhinderung von Übelkeit und Erbrechen $20 \mathrm{mg}$ Motilium als Tablette verabreicht. An sonstigen Nebenwirkungen können auftreten:

D-Cycloserin: Fieber, allergische Hauterscheinungen, Verdauungsstörungen, Übelkeit, Durchfall, Blutbildveränderungen, Leberschäden, Kopfschmerzen, Muskelzittern oder -Zuckungen, Sprachstörungen, Empfindungsstörungen, epileptische Anfälle, Müdigkeit, Schlaflosigkeit, Nervosität, Angst, Depression, Verwirrtheit, Trugwahrnehmungen, Gedächtnisstörungen, Euphorie, Sehstörungen

Pergolid: Bewegungsstörungen, Trugwahrnehmungen, Verwirrtheit, Schwindel, Schläfrigkeit, Schlaflosigkeit, Appetitlosigkeit, Übelkeit, Erbrechen, Verstopfung, Durchfall, Nasenlaufen, Atmungserschwernis, Blutdruckerniedrigung, kurzfristige Bewusstlosigkeit, unregelmäßiger Herzschlag, Sehen von Doppelbildern.

Domperidon: Bewegungsstörungen, Darmkrämpfe, allergische Reaktionen.

Die Studie umfasst voraussichtlich 9 Termine von je ca. 2 Stunden Dauer. Wir streben an, dass jeder Proband/jede Probandin alle Untersuchungen durchläuft. Selbstverständlich ist es Ihnen allerdings jederzeit möglich, ohne Angabe von Gründen und ohne dass Ihnen hieraus Nachteile entstehen von der Teilnahme an der Studie zurückzutreten. 
Für Ihre Teilnahme an der Studie wird Ihnen eine Aufwandsentschädigung von $7 € / \mathrm{h}$ bezahlt. Bei vorzeitigem Abbruch der Teilnahme erfolgt eine anteilige Auszahlung der Aufwandsentschädigung. Nachhaltige Wirkungen auf Ihre Erkrankung werden durch diese Untersuchung nicht angestrebt und sind auch nicht zu erwarten.

Falls einer der folgenden Punkte auf Sie zutrifft, ist eine Teilnahme an der Studie nicht möglich:

1. Herzschrittmacher

2. Metallimplantate im Kopfbereich (in den Kopfbereich eingesetztes Metall, z.B. Clips nach Operation eines intrazerebralen Aneurysmas (Gefäßaussackung im Bereich der Gehirngefäße), Implantation eine künstlichen Hörschnecke, eventuell auch Metallimplantate (Nägel, Platten, Schrauben) in anderen Körperabschnitten

3. Alter $<18$ oder $>60$ Jahre.

4. Hinweise auf eine chronische oder Residuen (Reste) einer neurologischen Erkrankung (Erkrankung des Nervensystems) in der Vorgeschichte.

5. Intrazerebrale Ischämien (Mangeldurchblutung des Gehirns/Schlaganfall/Blutungen) in der Vorgeschichte.

6. Hinweise auf epileptische Anfälle in der Vorgeschichte.

7. Vorliegen einer gravierenden internistischen (Erkrankung der inneren Organe) oder psychiatrischen (seelische Erkrankung) Vorerkrankung, insbesondere Schizophrenie (seelische Erkrankung mit Wahn, Trugwahrnehmungen und Störungen des Denkens) oder Manie (Erkrankung des Gemütes mit gehobener Stimmung).

8. Schwangerschaft oder Stillperiode

9. Drogen-, Medikamenten- und/oder Alkoholabhängigkeit

10. Rezeptive oder globale Aphasie (Störung des Sprachverständnisses bzw. zusätzlich des Sprechens)

Die aus der Studie gewonnenen Daten werden anonymisiert wissenschaftlich ausgewertet. Alle Erfordernisse des Datenschutzes werden dabei beachtet. Die möglichen Risiken sind als sehr gering einzustufen, dennoch wurde eine Probandenversicherung abgeschlossen. Sollten Sie als Folge der Studie Gesundheitsschädigungen erleiden, bitten wir Sie, uns dies unverzüglich mitzuteilen, damit wir dies der Versicherung melden können.

PD Dr. rer. nat. Andrea Antal

Abteilung Klinische Neurophysiologie
Dr. med. Michael A. Nitsche

Abteilung Klinische Neurophysiologie

Professor Dr. med. Walter Paulus

Abteilung Klinische Neurophysiologie 
Zentrum Neurologie

Abteilung Neurologie (Prof. Dr. Bähr)

Abteilung Klinische Neurophysiologie (Prof. Dr. W. Paulus)

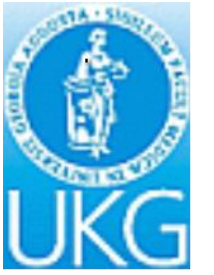

Abt. Klin. Neurophysiologie Leiter: Prof. Dr. W. Paulus

Robert-Koch-Str. 40

37075 Göttingen

Telefon: +4955139-6650/52

Telefax: $+4955139-8126$

\section{Einverständniserklärung zur Teilnahme an der Untersuchung: Einfluss schwachen transkraniell applizierten Gleichstroms auf Schmerzschwellen und sensorische Diskriminationsschwellen}

Frau/Herr Dr. hat mir heute anhand der Hinweise auf dem Informationsbogen für Teilnehmerinnen und Teilnehmer an der o.g. Studie die Durchführung der Untersuchung erläutert. Ich habe den Aufklärungstext gelesen und verstanden. Ich hatte die Möglichkeit, Fragen zu stellen, und habe die Antworten verstanden und akzeptiere sie. Alle mich interessierenden Fragen wurden ausreichend beantwortet. Mein Arzt hat mich über die mit der Teilnahme an der Studie verbundenen Risiken und den möglichen Nutzen informiert.

Ich erkläre darüber hinaus, dass ich alle Angaben zur Krankengeschichte wahrheitsgemäß gemacht habe.

Mir ist bekannt, dass ich jederzeit ohne Angabe von Gründen die weitere Untersuchung ablehnen bzw. meine Einverständniserklärung widerrufen kann, ohne dass mir daraus Nachteile entstehen. Ferner ist mir bekannt, dass ich auch mein Einverständnis zur Speicherung personenbezogener Daten widerrufen kann, ohne dass mir daraus Nachteile entstehen.

Ich bin bereit, an der Studie teilzunehmen.

Ich bin damit einverstanden, dass Daten, welche sich aus der Studie ergeben, ohne Erwähnung meiner Identität anonym veröffentlicht oder an zuständige Behörden weitergeleitet werden. Alle Befunde sind vertraulich und werden unter strikter Einhaltung des Datenschutzes gehandhabt.

Eine Kopie dieser Erklärung wurde mir überreicht.

Göttingen, den

Name der/des aufklärenden Ärztin/Arztes (in Druckbuchstaben) und Unterschrift

Name der/des Patientin/Patienten (in Druckbuchstaben) und Unterschrift 


\section{Danksagung}

Ich möchte mich an dieser Stelle sehr herzlich bei meiner Doktormutter Frau PD Dr. rer. nat. Andrea Antal bedanken, die mir von Anfang an immer Unterstützung und Hilfe bei der Erstellung dieser Arbeit war. Sowohl bei der Durchführung der Versuche als auch beim Schreiben beantwortete sie stets geduldig, freundlich und schnell meine Fragen und hatte immer ein offenes Ohr für etwaige Probleme.

Bei Daniella Terney, Leila Chaieb, Klára Boros und Csaba Poreisz möchte ich mich ebenfalls für ihre immer vorhandene Hilfsbereitschaft und ihre Ratschläge bedanken.

Dank sagen möchte ich auch Herrn Prof. Dr. Paulus als Leiter der Abteilung. 


\section{$\underline{\text { Lebenslauf }}$}

Ich wurde am 8.10.1982 in Detmold geboren und besuchte dort die Grundschule und das Gymnasium, das ich im Sommer des Jahres 2002 mit dem Abitur abschloss. Im Oktober 2002 begann ich mein Humanmedizinstudium an der Georg-August-Universität Göttingen. Das Physikum bestand ich am 13. September 2004, die ärztliche Prüfung am 19. Juni 2009.

Im April des Jahres 2005 begann ich meine Dissertation in der Abteilung für klinische Neurophysiologie im Zentrum für neurologische Medizin.

Während des Studiums famulierte ich in Gynäkologie in Berlin, in der chirurgischen Notaufnahme des Universitätsklinikums in Göttingen, als Stipendiatin der Ippnw in Chirurgie und Public Health am Universitätsklinikum in Izmir in der Türkei und in Pädiatrie an einem kleinen peruanischen Krankenhaus.

Mein praktisches Jahr absolvierte ich in Gynäkologie im St. Bernward Krankenhaus in Hildesheim, in Chirurgie am Krankenhaus der Universität Complutense in Madrid und in Innerer Medizin im Krankenhaus Links der Weser in Bremen. 